\title{
A Single Bolus of Docosahexaenoic Acid Promotes Neuroplastic Changes in the Innervation of Spinal Cord Interneurons and Motor Neurons and Improves Functional Recovery after Spinal Cord Injury
}

\author{
Zhuo-Hao Liu, ${ }^{1,2}$ Ping K. Yip, ${ }^{1}{ }^{\circ}$ Louise Adams, ${ }^{1}$ Meirion Davies, ${ }^{1}$ Jae Won Lee, ${ }^{1}$ Gregory J. Michael, ${ }^{1}$ John V. Priestley, \\ and Adina T. Michael-Titus ${ }^{1}$ \\ ${ }^{1}$ Queen Mary University of London, Barts and The London School of Medicine and Dentistry, Blizard Institute, London, E1 2AT, United Kingdom, and \\ ${ }^{2}$ Chang Gung Medical College and University, Chang Gung Memorial Hospital, Department of Neurosurgery, Linkou, Taiwan, 33305
}

Docosahexaenoic acid (DHA) is an $\omega$-3 polyunsaturated fatty acid that is essential in brain development and has structural and signaling roles. Acute DHA administration is neuroprotective and promotes functional recovery in animal models of adult spinal cord injury (SCI). However, the mechanisms underlying this recovery have not been fully characterized. Here we investigated the effects of an acute intravenous bolus of DHA delivered after SCI and characterized DHA-induced neuroplasticity within the adult injured spinal cord. We found robust sprouting of uninjured corticospinal and serotonergic fibers in a rat cervical hemisection SCI model. A mouse pyramidotomy model was used to confirm that this robust sprouting was not species or injury model specific. Furthermore, we demonstrated that corticospinal fibers sprouting to the denervated side of the cord following pyramidotomy contact V2a interneurons. We also demonstrated increased serotonin fibers and synaptophysin in direct contact with motor neurons. DHA also increased synaptophysin in rat cortical cell cultures. A reduction in phosphatase and tensin homolog (PTEN) has been shown to be involved in axonal regeneration and synaptic plasticity. We showed that DHA significantly upregulates miR-21 and downregulates PTEN in corticospinal neurons. Downregulation of PTEN and upregulation of phosphorylated AKT by DHA were also seen in primary cortical neuron cultures and were accompanied by increased neurite outgrowth. In summary, acute DHA induces anatomical and synaptic plasticity in adult injured spinal cord. This study shows that DHA has therapeutic potential in cervical SCI and provides evidence that DHA could exert its beneficial effects in SCI via enhancement of neuroplasticity.

Key words: docosahexaenoic acid; neuroplasticity; omega-3 polyunsaturated fatty acids; spinal cord injury

Significance Statement

In this study, we show that an acute intravenous injection of docosahexaenoic acid (DHA) 30 min after spinal cord injury induces neuroplasticity. We found robust sprouting of uninjured corticospinal and serotonergic fibers in a rat hemisection spinal cord injury model. A mouse pyramidotomy model was used to confirm that the robust sprouting involved V2a interneurons. We show that DHA significantly upregulates miR-21 and phosphorylated AKT, and downregulates phosphatase and tensin homolog (PTEN), which is involved in suppressing anatomical plasticity, in corticospinal neurons and in primary cortical neuron cultures. We conclude that acute DHA can induce anatomical and synaptic plasticity. This provides direct evidence that DHA could exert its beneficial effects in spinal cord injury via neuroplasticity enhancement.

\section{Introduction}

Docosahexaenoic acid (DHA) is an $\omega$-3 polyunsaturated fatty acid which is highly enriched in CNS membranes and plays an essential role in brain development (Salem et al., 2001). In animal models of Alzheimer's disease (Hashimoto et al., 2002; Lim et al., 2005) and traumatic brain injury (Wu et al., 2004; Bailes and Mills, 2010; Russell et al., 2013; Begum et al., 2014; Desai et al.,

This work was supported by Chang Gung Memorial Hospital, Taiwan CMRPG3A1051-1054 to Z.-H.L., CMDRP and Barts and the London Charity to P.K.Y. and A.T.M.-T., and the Nathalie Rose Barr PhD Studentship ISRT to L.A. and J.V.P. We thank Prof. F.J. Alvarez for advice on locomotor interneuron markers and Dr. L.-F. Wong for advice on qRT-PCR. 
2014), DHA can improve learning and memory. DHA has also been shown to induce significant functional improvements following spinal cord injury (SCI) (King et al., 2006; Huang et al., 2007; Figueroa et al., 2013; Lim et al., 2013) and ischemic injury (Pan et al., 2009; Eady et al., 2012). Various cellular mechanisms and processes have been proposed to mediate the effects of DHA. These include modulation of synaptic membrane fluidity and function (Jump, 2002), neurite extension (Calderon and Kim, 2004; Robson et al., 2010), gene expression (Duplus et al., 2000; Salem et al., 2001; Puskás et al., 2003), and synthesis of phosphatides in synaptic membranes (Wurtman et al., 2006). However, the full repertoire of underlying mechanisms remains to be determined.

SCI is a devastating condition that causes permanent disabilities related to the segmental level of injury. It is accompanied by pain and sensory loss, and currently there is no effective treatment. However, rehabilitative training can be beneficial (Wang and Sun, 2011; Fouad and Tetzlaff, 2012). This strategy is reliant on some degree of spontaneous recovery that occurs, particularly after incomplete spinal injuries (Tetzlaff et al., 2009). This recovery could involve adaptation of spinal central pattern generators and/or plastic changes of spinal pathways spared by the lesion (Raineteau and Schwab, 2001; Edgerton et al., 2004; Gulino et al., 2007). This has led to the concept that CNS axons have the ability to form new synaptic connections following denervation, and that this plays a key role in neural repair following CNS injury.

However, the degree of spontaneous recovery is limited, and new strategies need to be developed to enhance the process of plasticity to maximize the potential for recovery. Currently, a few studies focusing on stimulating plasticity with pharmacological intervention or rehabilitation programs have demonstrated some success (Ichiyama et al., 2008; García-Alías et al., 2009).

In the present study, we applied a single bolus DHA treatment in a rat cervical hemisection model to investigate the impact of this fatty acid on neuroplasticity in terms of functional and histological outcomes. Furthermore, a mouse pyramidotomy model was used to confirm the neuroplasticity observed, in another species and injury model. Primary neuronal cultures were used to further confirm the observations made in the in vivo studies, regarding anatomical and synaptic plasticity. The findings of this study indicate that a single bolus DHA treatment modulates neuroplasticity, both anatomical and synaptic, in two adult rodent spinal injury animal models. Combined with the neuroprotective effect of DHA observed in previous studies, this makes DHA a very promising candidate for the clinical treatment of SCI and traumatic brain injury.

\section{Materials and Methods}

Animal models. All animal research was performed under the United Kingdom Animals (Scientific Procedures) Act of 1986. Surgery was performed under anesthesia, and pain relief was provided appropriately during postoperative care.

Cervical hemisection in rat. Lateral cervical hemisection was performed in adult female Sprague Dawley rats (250-300 g, $n=5$ or 6 per group) using methods adapted from a previous study (King et al., 2006). Briefly, animals were anesthetized with isoflurane, and a dorsal midline incision was made at cervical level, to expose the vertebral laminae C4-C6. A left

The authors declare no competing financial interests.

Correspondence should be addressed to Prof. Adina T. Michael-Titus, Centre for Neuroscience and Trauma, Blizard Institute, Barts and The London School of Medicine and Dentistry, 4 Newark Street, London, E1 2AT, UK. E-mail: a.t.michael-titus@qmul.ac.uk.

DOI:10.1523/JNEUROSCI.0605-15.2015

Copyright $\odot 2015$ the authors $\quad 0270-6474 / 15 / 3512734-20 \$ 15.00 / 0$ hemi-laminectomy was performed at $\mathrm{C} 4$ and $\mathrm{C} 5$; then a cut between $\mathrm{C} 4$ and $\mathrm{C} 5$ was made with a microblade. Sham animals received only hemilaminectomy to expose the spinal cord without injury. After surgery, the muscles and skin layers were sutured and animals were returned to a warm incubator for recovery. Thirty minutes after hemisection, animals received a tail vein injection of either vehicle $(0.2 \%$ ethanol in saline) or DHA (Sigma D2534, $250 \mathrm{nmol} / \mathrm{kg}$ ) in a volume of $5 \mathrm{ml} / \mathrm{kg}$. The DHA dose chosen was based on previous studies from our laboratory showing functional improvements after SCI (King et al., 2006; Huang et al., 2007). Postoperative care involved subcutaneous injection of analgesic (buprenorphine, $0.01 \mathrm{mg} / \mathrm{kg}$ ) and saline twice daily for $3 \mathrm{~d}$ following surgery. To study the acute effect of DHA on PTEN levels, animals received cervical lateral hemisection before treatment with DHA or vehicle, as above. One day after injury, animals were perfused for histological analysis.

Pyramidotomy in the mouse. Right pyramidotomy was performed on adult female CD1 mice ( $n=5$ or 6 per group) using methods adapted from previous studies (Starkey et al., 2005; Yip et al., 2010). Briefly, mice were anesthetized with a mixture of medetomidine $(0.5 \mathrm{mg} / \mathrm{kg})$ and ketamine $(75 \mathrm{mg} / \mathrm{kg})$, and sterile precautions were used throughout. A ventral midline incision was made, and the surface of the occipital bone was exposed. The ventrocaudal part of the bone was partially removed using forceps before the right pyramidal tract was incised with iridectomy scissors. After surgery, the muscles and skin layers were sutured and animals returned to a warm incubator for recovery. Thirty minutes after pyramidotomy, animals received a tail vein injection of either vehicle $(0.2 \%$ ethanol in saline) or DHA ( $500 \mathrm{nmol} / \mathrm{kg}$ ) in a volume of $5 \mathrm{ml} / \mathrm{kg}$. Postoperative care involved subcutaneous injection of analgesic (buprenorphine, $0.01 \mathrm{mg} / \mathrm{kg}$ ) and saline twice daily for $3 \mathrm{~d}$ following surgery.

Behavioral testing. All behavioral testing and data analysis were performed blind by the experimenter.

Open field locomotion. The impairments in forelimb and hindlimb locomotor skills after cervical hemisection were evaluated using the forelimb locomotor scale (Cao et al., 2008) and the Basso, Beattie, Bresnahan (BBB) locomotor rating scale (Basso et al., 1995), respectively. Forelimb and hindlimb functions were evaluated in an open field measuring $\sim 1 \mathrm{~m}$ diameter and were observed for 4 min intervals. The open field locomotor tests were initially conducted daily for 2 weeks after surgery, then every other day until the end of the study.

Staircase test. The impairment in skilled left forelimb motor function after cervical hemisection was determined using the Montoya staircase test (Montoya et al., 1991; Yip et al., 2010). Animals were trained on the Montoya staircase test daily for $14 \mathrm{~d}$ before surgery, then tested daily for 1 week and thereafter every other day after injury. To either train or test the animals, each rat was placed into a Montoya staircase apparatus with each side of the stairs containing a food pellet per step, and left undisturbed for $20 \mathrm{~min}$ to perform the task. Thereafter, the numbers of food pellets displaced or eaten from each side of the stairs were recorded.

Grid exploration test. Deficits in voluntary descending motor control can be examined by assessing the ability of mice and rats to precisely control and place their forepaw and hindpaws onto grid wires (Starkey et al., 2005; Yip et al., 2010). A misplacement is recorded when the limb protrudes entirely through the grid and extends below the wire surface. All animals were tested for their baseline performance by subjecting them to the grid exploration test 3 times before surgery. The postsurgery testing was performed weekly until the end of the study.

Anterograde tracing. To evaluate whether the treatment had promoted axonal sprouting, we performed anterograde tracing of the intact corticospinal tracts (CST) as previously described (Yip et al., 2006, 2010). Tracing occurred 1 week after the hemisection in rats and 4 weeks after pyramidotomy in mice. Animals were placed in a stereotaxic frame, and burr holes were made into the skull ipsilaterally to the hemisection and contralaterally to the pyramidotomy. In the hemisected rats, burr holes were made at the following coordinates, defined as anteroposterior (AP) and mediolateral (ML): (1) AP: $-1.5 \mathrm{~mm}$, ML: $2.5 \mathrm{~mm}$; (2) AP: -0.5 mm, ML: $3.5 \mathrm{~mm}$; (3) AP: $-0.5 \mathrm{~mm}$, ML: $2.5 \mathrm{~mm}$; (4) AP: $0.5 \mathrm{~mm}$, ML: $3.5 \mathrm{~mm}$; (5) AP: $1.0 \mathrm{~mm}$, ML: $1.5 \mathrm{~mm}$; and (6) AP: $1.5 \mathrm{~mm}, \mathrm{ML}: 2.5 \mathrm{~mm}$; and (7) AP: $2.0 \mathrm{~mm}, \mathrm{ML}: 3.5 \mathrm{~mm}$, relative to bregma. In the pyramidotomy model, the holes were made at the following coordinates: (1) AP: $-1.0 \mathrm{~mm}$, L: $0.5 \mathrm{~mm}$; (2) AP: $-1.0 \mathrm{~mm}$, L: $1.0 \mathrm{~mm}$; (3) AP: $-0.5 \mathrm{~mm}, \mathrm{~L}$ : 
$1.0 \mathrm{~mm}$; (4) AP: $-0.5 \mathrm{~mm}$, L: $0.5 \mathrm{~mm}$; (5) AP: $0 \mathrm{~mm}$, L: $1.0 \mathrm{~mm}$; (6) AP: 1.0 mm, L: 1.0 mm; (7) AP: 1.5 mm, L: 1.0 mm; (8) AP: 2.0 mm, L: 1.0 $\mathrm{mm}$. At each site, injections of biotinylated dextran amine (BDA; $10 \%$; $10,000 \mathrm{MW}, 1 \mu \mathrm{l} / \mathrm{site}$ for rat and $0.2 \mu \mathrm{l} /$ site for mouse) were delivered using a glass micropipette attached to a Hamilton syringe via water-filled polyethene tubing. The micropipette was inserted below the skull surface ( $2 \mathrm{~mm}$ deep for rat and $1 \mathrm{~mm}$ for mouse) and BDA delivered at a rate of $0.2 \mu \mathrm{l} / \mathrm{min}$. Animals were subsequently maintained for 2 weeks before tissue was collected for histology.

Histology. At the appropriate postinjury interval, animals were deeply anesthetized with sodium phenobarbital $(80 \mathrm{mg} / \mathrm{kg}$ i.p.) and perfused with $0.9 \%$ saline followed by $4 \%$ paraformaldehyde (PFA) in $0.1 \mathrm{M}$ phosphate buffer. The brain and spinal cord were dissected out, postfixed in $4 \%$ PFA for $2 \mathrm{~h}$, and cryoprotected in $20 \%$ sucrose in $0.1 \mathrm{M}$ phosphate buffer at $4^{\circ} \mathrm{C}$ until further processed. The tissues were embedded in OCT medium before cutting using a cryostat. The rat cervical spinal cords containing C3-C7 levels were sectioned $(15 \mu \mathrm{m})$ serially in the horizontal plane and placed onto 25 Superfrost slides, which provided 5 spinal cord sections per slide. The cervical region (C3-C7) of the pyramidotomy mice was sectioned $(15 \mu \mathrm{m})$ serially in the transverse plane. The rat brains containing the sensorimotor cortex region were sectioned (20 $\mu \mathrm{m})$ serially in the coronal plane.

Randomly selected slides of brain and spinal cord sections were processed for immunohistochemistry using the following primary antibodies: rabbit anti-serotonin antibody (1:4000, Immunostar) to examine serotonin axons (Ramer et al., 2004), rabbit anti-synaptophysin antibody (1:1000, Cell Signaling Technology) to examine synaptic boutons (Averill et al., 2004), goat anti-choline acetyltransferase (ChAT, 1:100, Chemicon) to examine cholinergic neurons, rabbit anti-protein kinase $\mathrm{C} \gamma$ (PKC $\gamma, 1: 500$, Santa Cruz Biotechnology) to examine the corticospinal tract, mouse anti-neuron-specific nuclear protein (NeuN, 1:1000, Chemicon) to examine mature neuronal cell bodies, rabbit anti-PTEN (1:300, Cell Signaling Technology) to examine a major negative regulator of the PI3K/Akt signaling pathway (Zhao et al., 2013), and sheep antiChx10 antibody (1:100, Abcam) to detect V2a group interneurons (AlMosawie et al., 2007; Dougherty and Kiehn, 2010). Sections were incubated in 10\% normal donkey or goat serum for $30 \mathrm{~min}$ followed by an overnight incubation with primary antibodies. Sections were washed three times ( $5 \mathrm{~min}$ each) in $10 \mathrm{~mm}$ PBS before being incubated for $2 \mathrm{~h}$ in the appropriate secondary antibodies conjugated to AlexaFluor- 488 or -594 (1:1000). After another three 5 min washes in PBS, sections were then counterstained with the fluorescent nuclear dye bis-benzimide (Hoechst 33342; $1 \mu \mathrm{g} / \mathrm{ml}$ PBS; Sigma) for $5 \mathrm{~min}$ to facilitate detection of cell nuclei or in NeuroTrace 435/455 Blue Fluorescent Nissl (1:100, Invitrogen) to stain the neuronal cell bodies. Slides were mounted in ProLong Gold antifade reagent.

The tyramide signal amplification technique was performed for detection of BDA-labeled corticospinal tract fibers and PTEN immunostaining in the cortex. Sections were washed three times ( 5 min each) in PBS and then incubated with $0.3 \%$ hydrogen peroxide for $30 \mathrm{~min}$. After 3 further 5 min washes with PBS, the sections were incubated in avidinbiotin-peroxidase complex (1:250 'A' and 1:250 'B' in PBS, prepared 30 min before use, Vectastain ABC Elite Kit, Vector Laboratories) for 30 $\mathrm{min}$ at room temperature. Following $3 \times 5$ min washes in PBS, sections were incubated with tyramide (1:75, NEN Life Sciences) for $10 \mathrm{~min}$. After further $3 \times 5 \mathrm{~min}$ washes in PBS, the sections were incubated with extra-avidin FITC (1:400) for $2 \mathrm{~h}$ before the sections were immunostained with another antibody for double-labeling or washed, mounted, and coverslipped.

In situ hybridization (ISH) was performed as described previously (Lopez-Ramirez et al., 2014), except for minor changes. Sections were first washed in PBS for 5 min for 3 times before proteinase K treatment 2 $\mu \mathrm{g} / \mathrm{ml}$ at $37^{\circ} \mathrm{C}$ for $10 \mathrm{~min}$. Sections were then fixed again for $5 \mathrm{~min}$ in $4 \%$ PFA and dehydrated in 70\%, 96\%, and 99.9\% ethanol twice for $1 \mathrm{~min}$ each time. Double digoxigenin-labeled miRcuryLNA probe miR-21 oligonucleotide ( $5 \mathrm{~nm}$; Exiqon) was hybridized with the sections $1 \mathrm{~h}$ at $53^{\circ} \mathrm{C}$. After hybridization, the sections were washed in a series of 5 min salinesodium citrate washes at $53^{\circ} \mathrm{C}$ and then incubated for $15 \mathrm{~min}$ in a blocking solution $(0.05 \%$ Tween and $1 \%$ sheep serum) before incubation overnight in sheep anti-digoxigenin antibody conjugated with alkaline phosphatase (Roche), diluted to a concentration of 1:800 in dilution buffer $(0.05 \%$ Tween, $1 \%$ sheep serum, and $1 \%$ BSA). Next, the sections were washed with TBST 3 times before incubation for $5 \mathrm{~min}$ twice in alkaline development buffer ( $100 \mathrm{~mm}$ Tris $\mathrm{HCl}, \mathrm{pH} 9.5 ; 100 \mathrm{~mm} \mathrm{NaCl} ; 50$ mM $\mathrm{MgCl}_{2}$; and $0.1 \%$ Tween 20), and then incubated with NBT/BCIP ( $1 \%$; Roche) and levamisole $\left(0.5 \%\right.$; Vector Laboratories), at $30^{\circ} \mathrm{C}$ overnight. Finally, sections were washed in KTBST ( $50 \mathrm{~mm}$ Tris $\mathrm{HCl}, \mathrm{pH} 7.5$, $150 \mathrm{~mm} \mathrm{NaCl}, 20 \mathrm{~mm} \mathrm{KCl}, 0.5 \%$ Tween 20 ) for $5 \mathrm{~min}$ to stop the reaction, then washed with water. Slides were dehydrated at room temperature overnight, then mounted with mounting medium.

Primary cortical neuronal cultures. Cortical tissue was dissected from embryonic Sprague Dawley rat brain (E18) in ice-cold HBSS and then incubated with $0.125 \%$ trypsin for $8 \mathrm{~min}$. After the trypsin solution digestion, FBS was added to inactivate remaining trypsin. Cells were mechanically dissociated; and after the cells were allowed to settle, the supernatant was discarded. Cells were resuspended in $1 \mathrm{ml}$ modified Neurobasal medium and filtered through a $70 \mu \mathrm{m}$ mesh. After washing and pelleting, cells were resuspended to a seeding density of $0.25 \times 10^{6}$ cells $/ \mathrm{ml}$ onto pre-poly-D-lysine-coated glass coverslips and incubated at $37^{\circ} \mathrm{C}$ in $5 \% \mathrm{CO}_{2}$.

Synaptogenesis study. At 1 days in vitro (DIV), DHA treatment ( 1 or 3 $\mu \mathrm{M}$ ) was applied to the cortical cultures. Every third day thereafter, half of the media was replaced with fresh media. At 14 DIV, cultures were fixed with $4 \%$ PFA for $20 \mathrm{~min}$, permeabilized with cold methanol, and washed with $3 \times 5$ min PBS as previously described (Yip et al., 2006). For immunostaining, cortical neuronal culture was incubated at room temperature for $2 \mathrm{~h}$ with primary antibodies: mouse anti-MAP2 (1:1000), rabbit antisynaptophysin (1:500). Secondary antibody staining was performed with a mixture of AlexaFluor-488 (1:1000, Invitrogen) and AlexaFluor-594 (1:1000, Invitrogen) at room temperature for $45 \mathrm{~min}$. After PBS washes, cells were mounted with FluorSave reagent (Calbiochem) and observed under a Zeiss microscope. The immunoreactivity of synaptophysin in various areas on the coverslip was measured. Ten images at $40 \times$ magnification were captured for synaptic bouton analysis. The first 10 neurons per image were analyzed for the number of synaptic boutons per cell. In total, 100 neurons were analyzed per treatment group per independent experiment. For the analysis of the percentage cell body contacted by synaptophysin boutons, a personally designed script was created in ImageJ, which highlights the cell body stained with MAP2 and then overlays the synaptophysin staining. The total area of synaptophysin staining was divided by the cell body area per neuron.

Inhibitory and proregenerative molecules study. To study the intracellular inhibitory and proregenerative molecules in the presence of DHA, cortical neurons at 1 DIV were treated with either vehicle (BS media with $0.01 \%$ ethanol) or DHA (1-3 $\mu \mathrm{M})$. After a further 2 DIV, the neurons were fixed and stained as above with primary antibodies: rabbit antiPTEN (D4.3, 1:200), rabbit anti-phospho-AKT (Ser 473, 1:100), and mouse anti- $\beta$-III tubulin (1:1000). To study the effect of DHA on the mTOR pathway, $30 \mathrm{~nm}$ rapamycin, an inhibitor of the mTOR signaling pathway, was added an hour before the application of DHA. Furthermore, to study the role of DHA on miR-21, 3 pmol/ml miRCURY LNA microRNA inhibitor specificially for rno-miR-21-5p (Exiqon) complexed with the transfection reagent Lipofectamine 2000 (Invitrogen) was added an hour before application of DHA. Neurite length was analyzed by drawing along every neurite per cell body using the ImageJ program. The maximum neurite length was quantified per neuron.

Western blot analysis. Western blots were performed as previously described (Yip et al., 2010). After 1 DIV, primary cortical neurons were treated with DHA and left for a further 2 DIV. Thereafter, media were removed and neurons harvested in $200 \mu$ ice-cold protein lysis buffer. Two wells of each treatment were pooled together and rotated for $2 \mathrm{~h}$ at $4^{\circ} \mathrm{C}$ before centrifugation at $13,500 \times g$ for $15 \mathrm{~min}$ at $4^{\circ} \mathrm{C}$. The protein level from the collected supernatant was determined using the bicinchoninic acid protein assay kit (Pierce). A total of $10 \mu \mathrm{g}$ of total protein mixed with loading buffer was electrophoresed on $12 \%$ acrylamide gel before transfer onto Hybond P membranes (GE Healthcare). Blots were blocked with 5\% skimmed milk for at least 30 min and then incubated overnight at room temperature and pressure with 

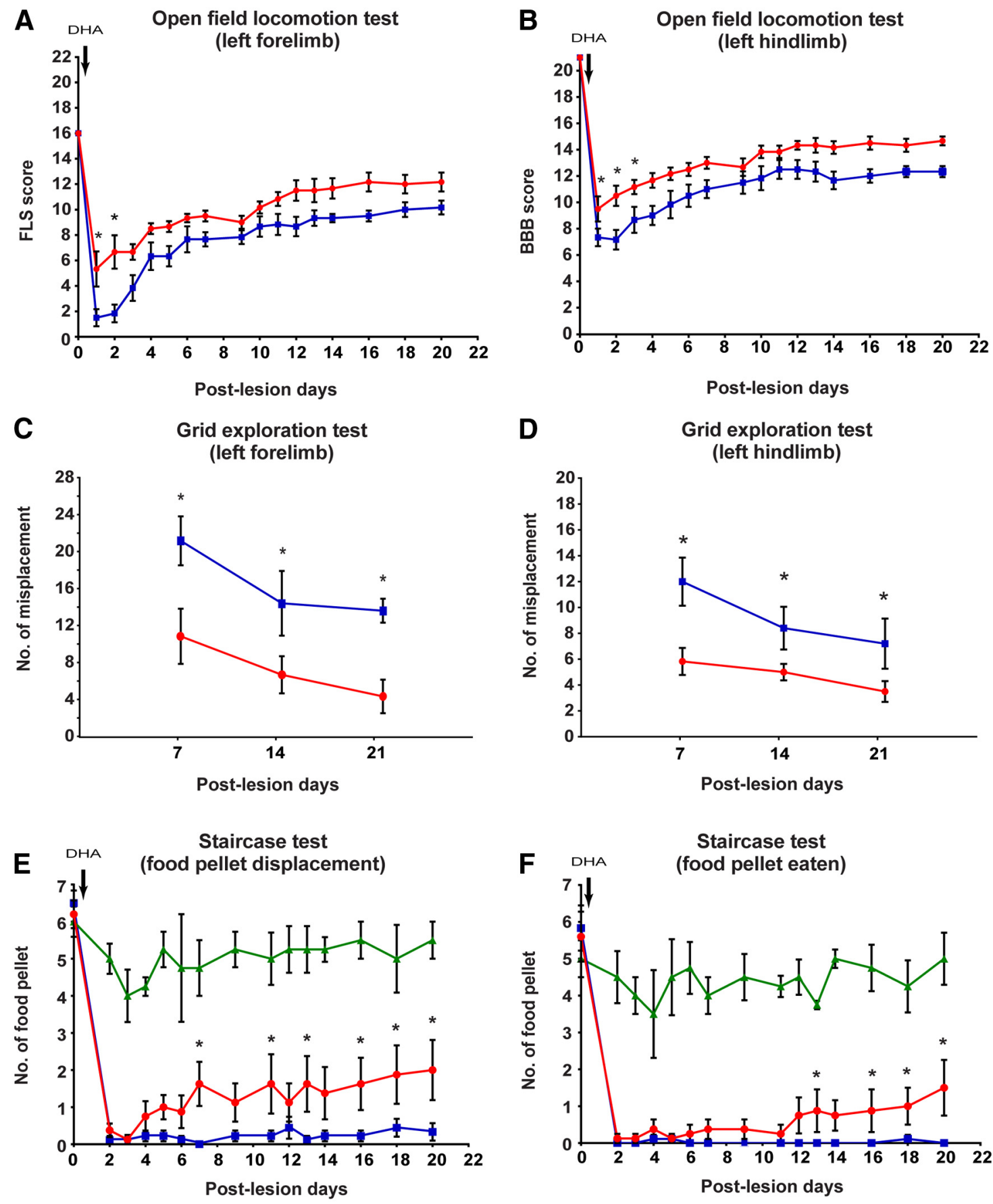

- DHA $\quad$ - Vehicle \pm Sham

Figure 1. DHA enhances functional recovery following cervical lateral hemisection in rat. $\boldsymbol{A}, \boldsymbol{B}, \mathrm{In}$ the open field locomotor test, DHA-treated animals (red circles) had significantly higher locomotor scale scores on the ratings of forelimb use than the vehicle group (blue squares) during the first and second day after surgery. The hindlimb BBB locomotor scores revealed a significant difference during the first, second, and third day after surgery. $\mathbf{C}, \boldsymbol{D}$, In the grid exploration test, DHA-treated animals (red circles) had fewer forelimb and hindlimb misplacements throughout the testing period compared with vehicle-treated animals (blue squares). $\boldsymbol{E}, \boldsymbol{F}$, In the Montoya staircase test, all injured animals lost the ability to displace (gross motor function) and eat (fine motor function) the food pellet after cervical lateral hemisection $2 \mathrm{~d}$ after injury. The animals treated with vehicle (blue squares) did not recover any food retrieval ability, but DHA-treated animals (red circles) gradually recovered food retrieval ability from around 2 weeks onwards compared with uninjured sham operated animals (green triangles). ${ }^{*} p<0.05$. Results represent mean \pm SEM.

primary antibodies: rabbit anti-phospho-AKT (Ser 473, 1:100) and rabbit anti-PTEN (D4.3, 1:200). Visualization was performed using goat anti-rabbit immunoglobulins/HRP (1:5000) for $1 \mathrm{~h}$, then followed by ECL Prime Western blotting detection reagent (GE Healthcare) for 5 min before exposure to Hyperfilm ECL (GE Healthcare).

To determine the total AKT as an internal control on the same blot, the blot was incubated with the Thermo Scientific Restore Plus Western blot stripping buffer for $5 \mathrm{~min}$, blocked with 5\% milk for $30 \mathrm{~min}$, and then incubated overnight with rabbit anti-total AKT (1:100). Western blotting was performed on three or four independent samples.

$q R T-P C R$ of microRNA. Cortical neuron cultures were prepared as mentioned above. MirVana PARIS isolation kit (Ambion, catalog \#AM1556) was used to extract total RNA from cortical neurons according to the manufacturer's instruction as previously described (Strickland et al., 2011). Total 

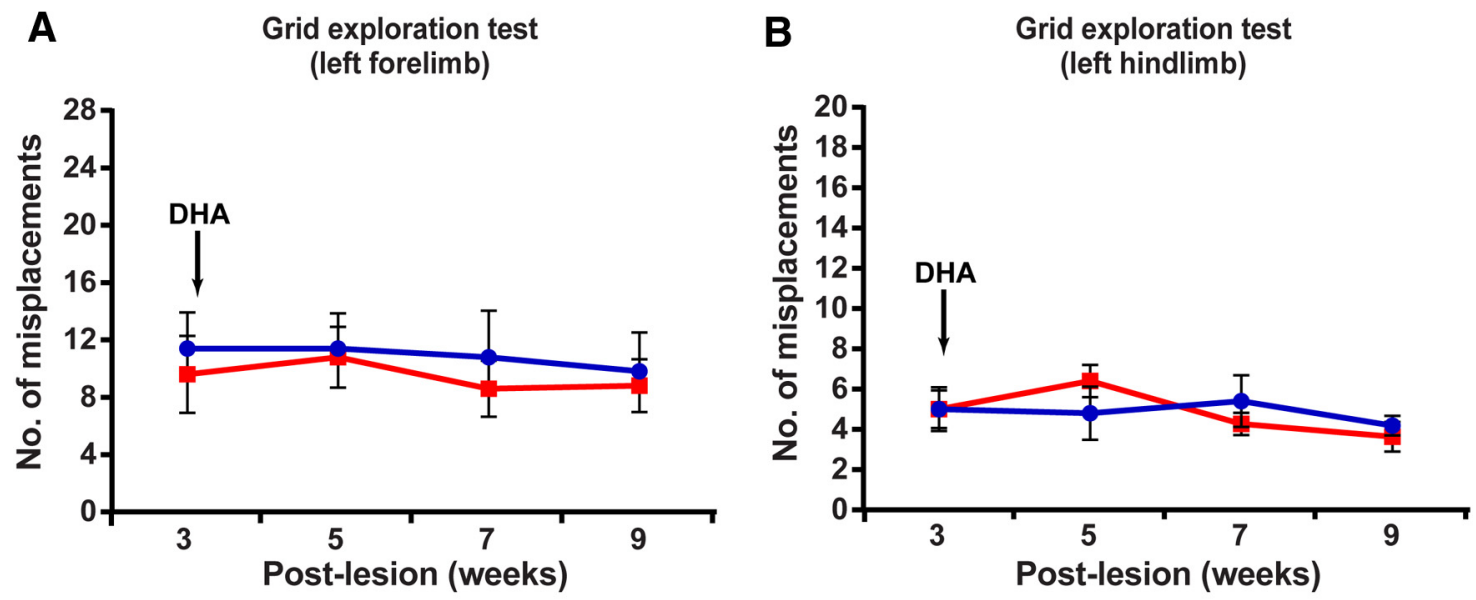

C Staircase test: food pellet displacement

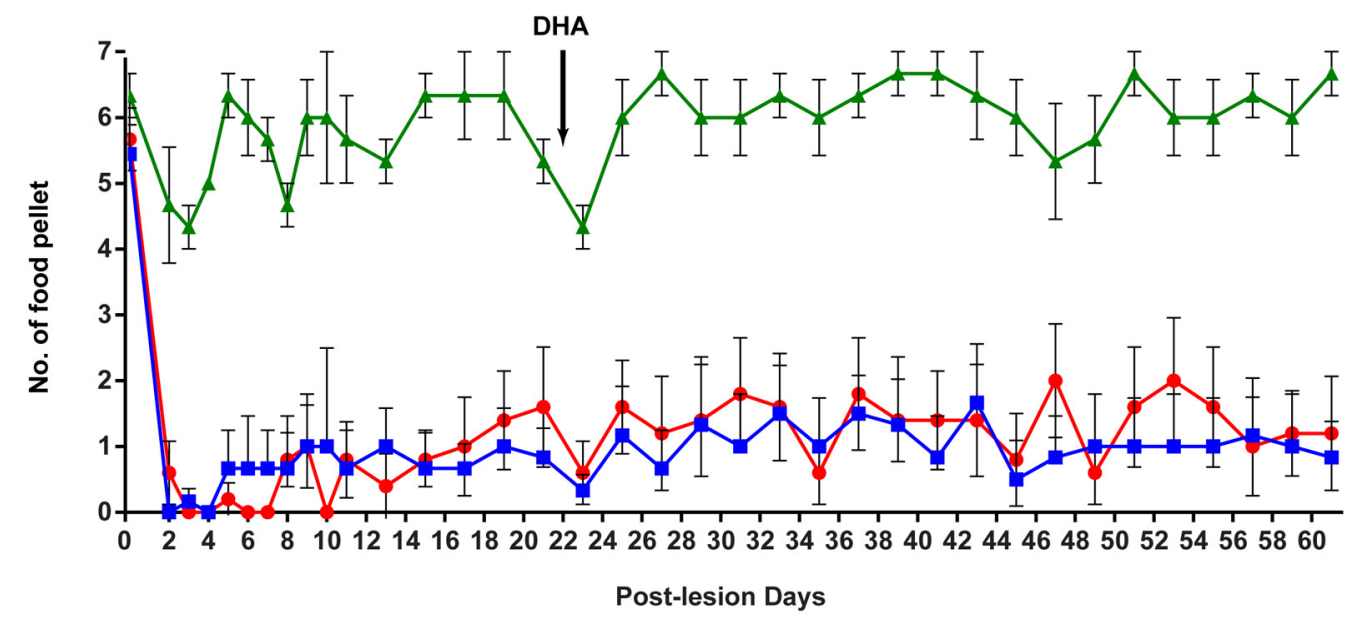

D Staircase test: food pellet eaten

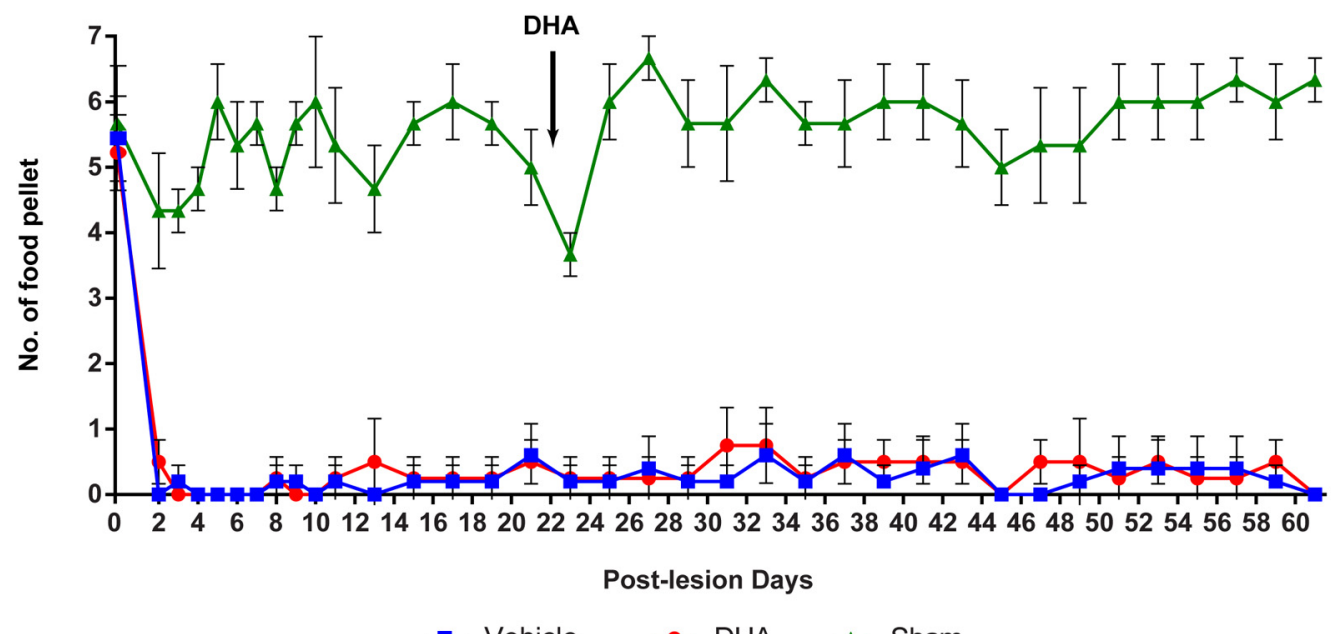

Figure 2. Delayed DHA treatment does not promote functional recovery when administered in the subacute phase of SCI. Delayed DHA treatment was administered 3 weeks after cervical spinal hemisection. In the grid exploration test, there are no significant differences in left forelimb $(\boldsymbol{A})$ and hindlimb $(\boldsymbol{B})$ misplacements between the vehicle and DHA-treated groups during the period of behavioral assessment. $\boldsymbol{C}, \boldsymbol{D}$, In the Montoya staircase test, all injured animals lost the ability to displace (gross motor function) and eat (fine motor function) the food pellet after cervical lateral hemisection, from $2 \mathrm{~d}$ after injury. The animals treated with either vehicle (blue square) or DHA (red circle) at 3 weeks after injury did not significantly recover food retrieval ability compared with uninjured sham operated animals. Results represent mean $\pm S E M ; n=5$ or 6 animals in each group. 
A
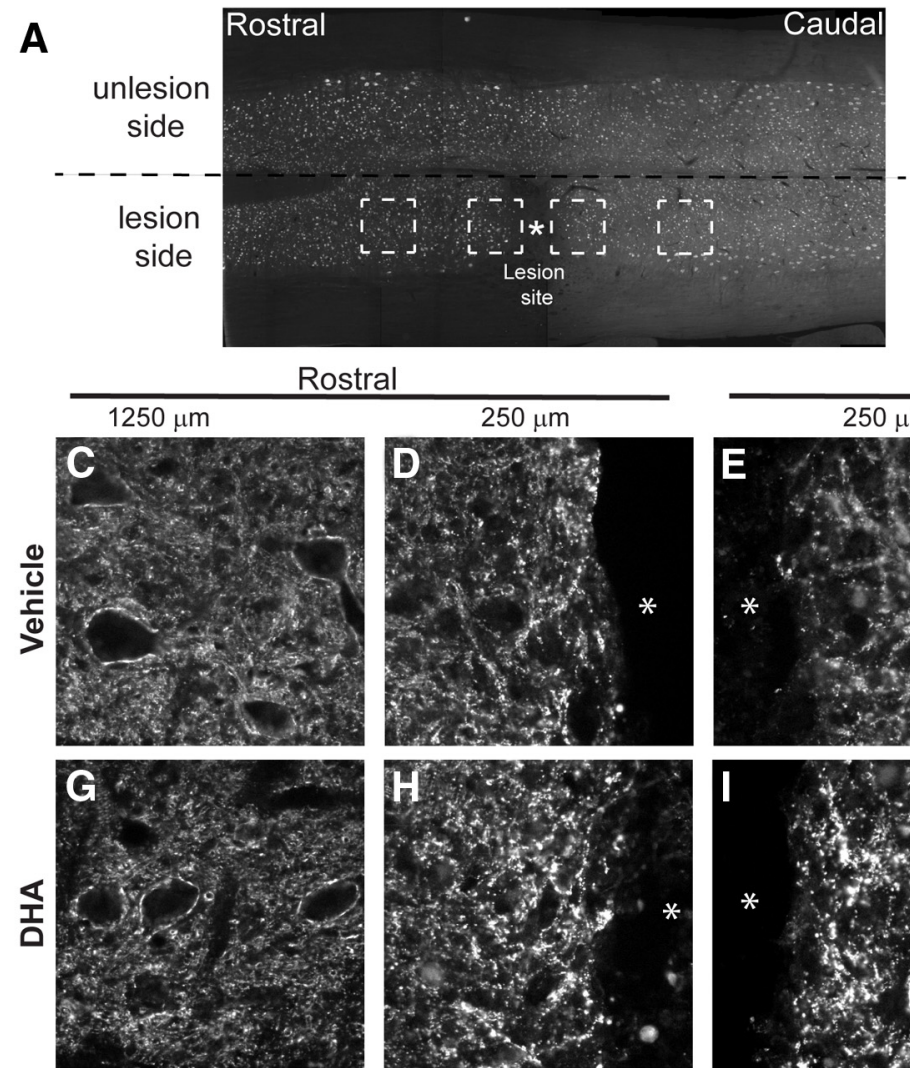

K

Rostral

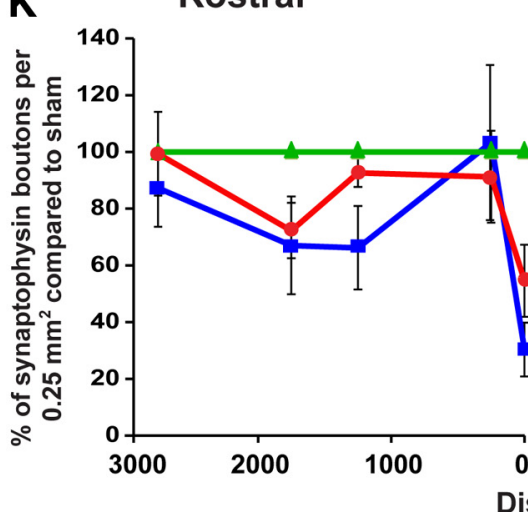

Distance from the lesion site $(\mu \mathrm{m})$
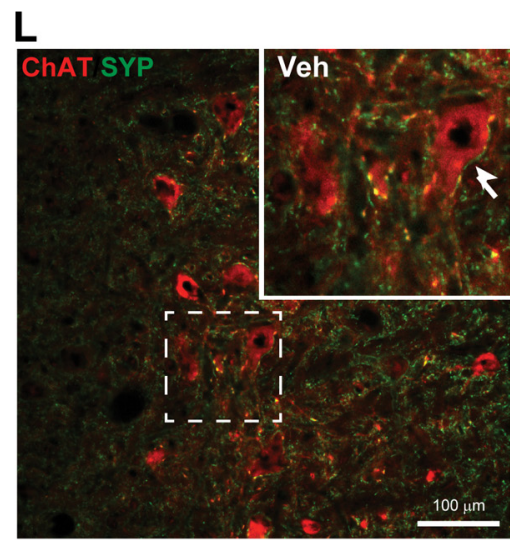

M

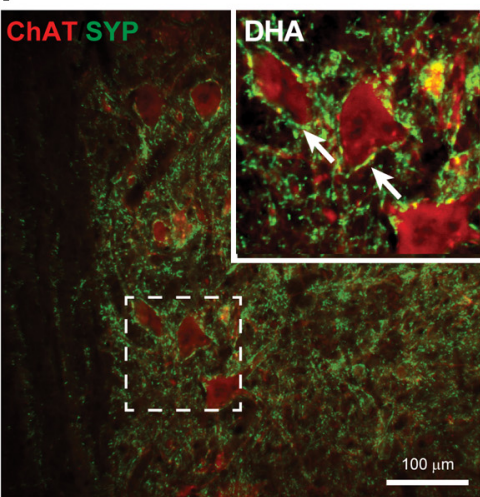

B

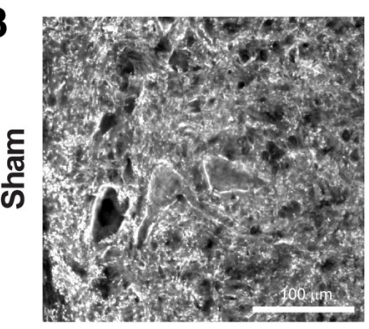

Caudal
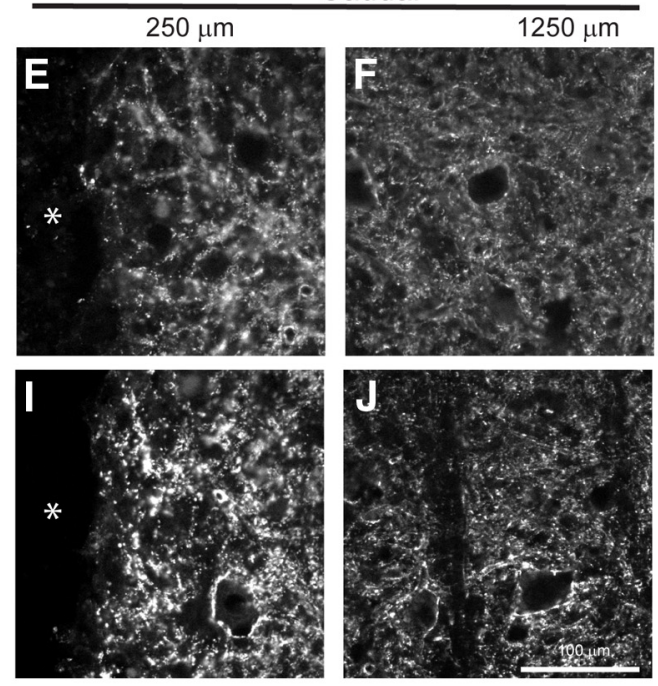

\section{Caudal}

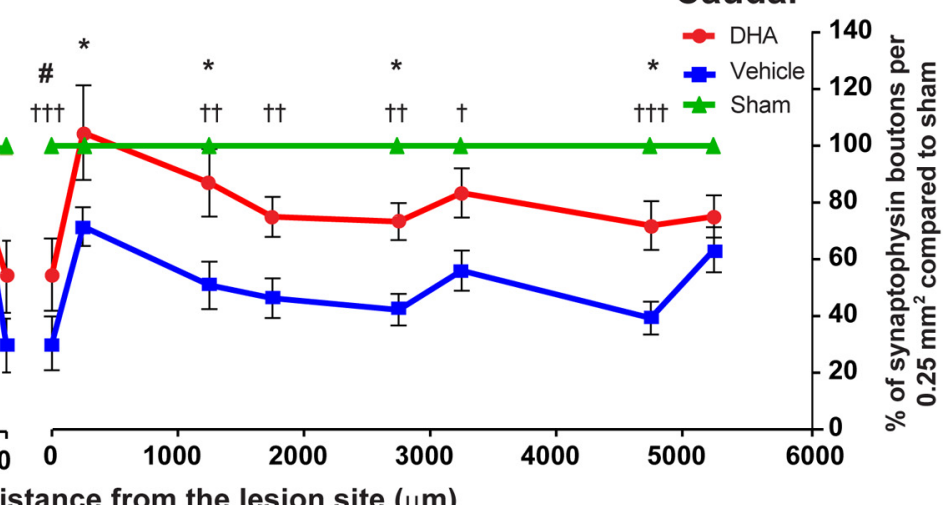

$\mathbf{N}$

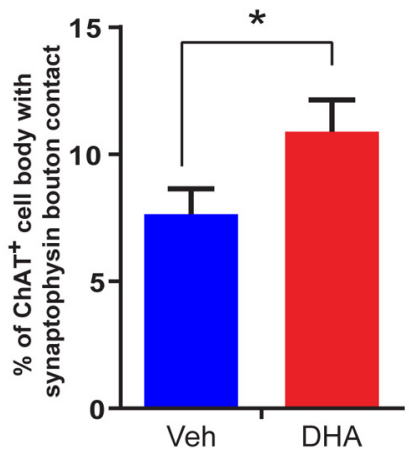

Figure 3. DHA enhances synaptophysin-immunopositive synaptic terminals after cervical hemisection in the rat. $\boldsymbol{A}$, Lower-magnification image of the cervical hemisection lesion and regions where photomicrographs and analysis were performed. $\boldsymbol{B}$, Photomicrograph showing immunolabeling of synaptophysin from the spinal cord of a sham operated animal. $\mathbf{C}-\boldsymbol{J}$, Photomicrographs showing immunolabeling of synaptophysin along the rostrocaudal plane in horizontal sections of the spinal cord of vehicle (top panels) and DHA-treated (bottom panels) rats following cervical lateral hemisection. Stars indicate the lesion site. $\boldsymbol{K}$, The analysis of synaptophysin immunoreactivity revealed significantly fewer synaptic terminals in the (Figure legend continues.) 
RNA concentration was quantified using an ND 1000 spectrophotometer (NanoDrop Technologies). qRT-PCR analysis of miR-21 expression was performed using TaqMan miRNA assays for miR-21 (assay ID: 000397) and RNU6B as internal control (assay ID: 001093) according to the manufacturer's instructions and performed on 7500 Real-Time PCR System (Applied Biosystems). Experiments were performed in triplicates and from five independent samples.

Image capture and analysis. Image analysis and quantification were performed with the observer blinded to the group assignment. At least 4 sections per staining of the corresponding plane containing the region of interest (e.g., motor neurons) were viewed and captured on a Leica epifluorescence microscope at $20 \times$ magnification. Selected sections were also examined on an LSM510 confocal microscope (Zeiss). Quantitative analysis of the number of serotonin-labeled axons, BDA-labeled corticospinal axons, and synaptophysin-labeled boutons was conducted by capturing images at various rostral and caudal levels versus the lesion site. Immunoreactivity in captured images was then counted in a $500 \mu \mathrm{m} \times$ $500 \mu \mathrm{m}$ measuring frame by using the ImageJ analysis program (ImageJ $1.46 \mathrm{u}$, National Institutes of Health). Images were converted to a set threshold and then counted automatically using customized macros. The level of immunoreactivity was expressed as the percentage of the area of the measuring frame that contained immunoreactivity.

Statistical analysis. The behavioral assessment and histological analysis were performed blind. All statistical analyses were performed using GraphPad Prism. The data were presented as mean \pm SEM. One-way, two-way, or two-way repeated-measures ANOVA was used to compare experimental groups, with Tukey's post hoc comparisons for equal sample sizes or Bonferroni's post hoc test for unequal sample sizes. Differences were considered significant when $p<0.05$.

\section{Results}

\section{A single DHA injection promotes functional recovery after} cervical hemisection

Hemisected rats treated with DHA showed significant improvements in both the forelimb locomotor scale and BBB score, already detectable at days 1 and 2 after injury compared with vehicle-treated animals, indicating better controlled limb movements after DHA treatment (Fig. $1 A, B$ ). Furthermore, significant improvement $3 \mathrm{~d}$ after injury was observed in the hindlimb locomotion using the BBB score, in the DHA-treated group compared with vehicle treatment (Fig. $1 B$ ). The improvements were sustained for the whole study duration. These results show that a single bolus injection of DHA induced significant functional improvement after cervical SCI, and this is similar to the functional improvement previously reported after a thoracic cord hemisection (King et al., 2006). To demonstrate functional use of both forelimb and hindlimbs, the animals' sensorimotor performance was tested using the grid exploration test. A significant reduction in misplacements was observed in DHA-treated animals in both forelimb and hindlimbs compared with vehicle-treated animals, at 7-21 d after injury (Fig. 1C,D). To fully demonstrate voluntary forelimb functionality and dexterity, animals were required to grasp food pellets to eat in the staircase test. DHA-treated animals were able to displace significantly more food pellets by $7 \mathrm{~d}$ after

\footnotetext{
$\leftarrow$

(Figure legend continued.) vehicle-treated group (blue circles) than the DHA-treated group (red squares), compared with the sham operated group (green triangles). $L, M$, Images represent the difference in density of contacts between synaptophysin-immunopositive terminals (green) and choline acetyltransferase-immunopositive motor neurons (red) in DHA- and vehicle-treated groups. $\boldsymbol{N}$, The analysis confirmed the increase in the density of synaptophysin contacts on ventral horn motor neurons in DHA-treated rats (red bar) compared with vehicletreated animals (blue bar). ${ }^{*} p<0.05$, vehicle versus DHA group. $\uparrow p<0.05$, vehicle versus sham group. $t+p<0.01$, vehicle versus sham group. $t+\dagger p<0.001$, vehicle versus sham group. ${ }^{\#} p<0.05$, DHA versus sham group. Scale bar, $100 \mu \mathrm{m}$. Results represent mean $\pm \mathrm{SEM} ; n=6$ animals per group.
}

injury compared with the vehicle-treated animals (Fig. 1E). Furthermore, at $13 \mathrm{~d}$ after injury, DHA-treated animals were able to grasp and eat significantly more food pellets than the control group (Fig. $1 F$ ). Improvement in both food pellets displaced and pellets eaten remained significant until the end of the study, $21 \mathrm{~d}$ after injury (Fig. $1 E, F$ ). Interestingly, a delayed single bolus DHA injection at 3 weeks after injury after cervical lateral hemisection did not promote significant functional recovery in either the grid exploration or the staircase tests (Fig. $2 A-D$ ). These data suggest that a single bolus injection of DHA administered early after injury, but not delayed, can induce sensorimotor and voluntary functional recovery after cervical injury.

\section{A single DHA injection promotes synaptogenesis}

To evaluate the effect of DHA on neuroplasticity in vivo, we studied the involvement of DHA in synaptic remodeling because this could support functional recovery. The effect of DHA on synaptic terminals after cervical lateral hemisection was analyzed using the synaptic vesicle protein, synaptophysin. In sham operated animals, there was extensive immunoreactivity for synaptophysin in the spinal cord (Fig. 3B). After cervical lateral hemisection, a significant decrease in synaptophysin immunostaining was observed caudal to the lesion site for the injured vehicle-treated group (Fig. 3C-J). There was a difference caudally from $250 \mu \mathrm{m}$ as far as $4750 \mu \mathrm{m}$ from the lesion site, compared with the sham animals (Fig. $3 \mathrm{~K}$ ). However, in DHA-treated animals, this decrease in synaptophysin immunostaining due to the injury was markedly reduced (Fig. $3 K$ ). A closer examination of synaptophysin-immunopositive boutons around individual ChAT-immunopositive motor neurons in the ventral horn was performed. The overall density of synaptophysin boutons in contact with motor neurons was significantly greater in the DHAtreated animals compared with the vehicle-treated animals (Fig. $3 L, M$ ).

To further evaluate the effect of DHA on synaptogenesis, primary embryonic cortical neuronal cultures were used to examine synapse development (Fig. 4A-F). Application of 1 and $3 \mu \mathrm{M}$ DHA resulted in significant increases in the numbers of synaptophysin boutons present around neuronal cells compared with the vehicle-treated cells (Fig. $4 F, G$ ), without significant alteration in the numbers of cortical neurons (Fig. 4H). This shows that DHA can enhance synaptogenesis in cortical neurons and specifically increase the number of synaptic terminals contacting neurons.

\section{A single DHA injection promotes axonal sprouting after cervical hemisection \\ Serotonin fibers}

To investigate in detail how DHA administration can promote functional recovery, and identify possible origins of the sprouting synaptophysin terminals, axonal sprouting of serotonin fibers rostrally and caudally to the lesion site was examined at 3 weeks after injury (Fig. 5A-H). In both injured groups, a marked increase in serotonin fibers was observed rostral to the lesion site. However, in the caudal region, in DHA-treated animals, there was a significant increase in serotonin fibers compared with vehicle-treated or sham animals (Fig. 5I). The significant increase in serotonin fibers in the caudal region was not in the vicinity of the lesion site but was distal, in a region between 1250 and $3250 \mu \mathrm{m}$ from the lesion site. Interestingly, in separate experiments, 1 week after cervical hemisection, an increase in serotonin fibers rostral, and a decrease caudal to the injury site, was seen (Fig. $5 J$ ). However, the numbers of serotonin fibers caudal to the lesion site had increased by 3 weeks after injury and were 
A

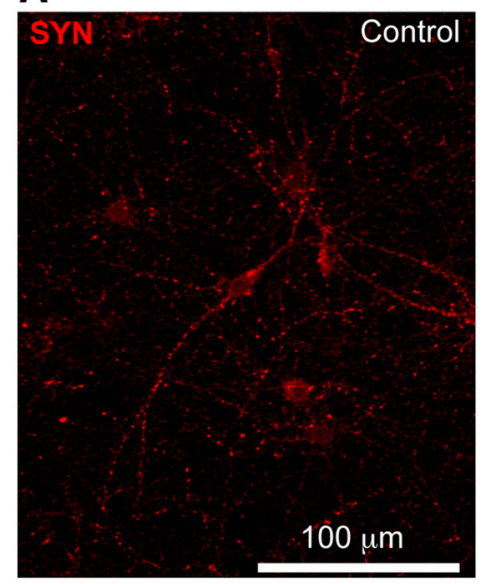

D

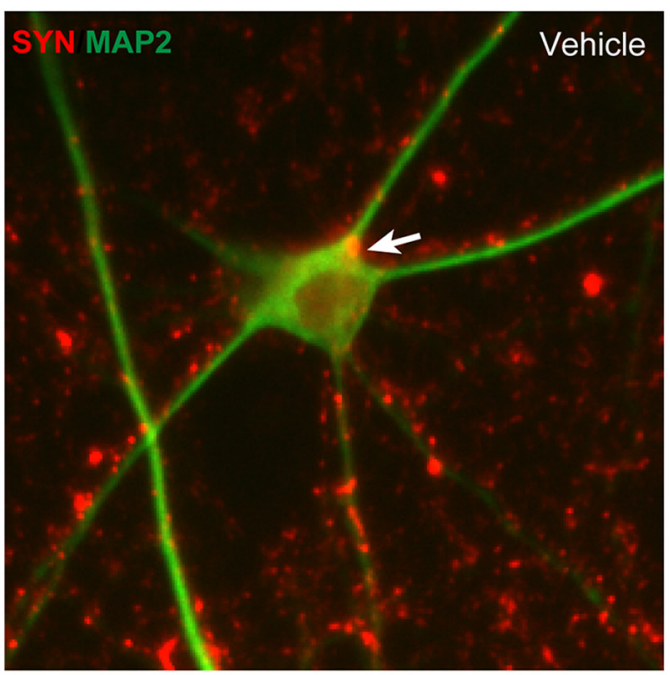

B

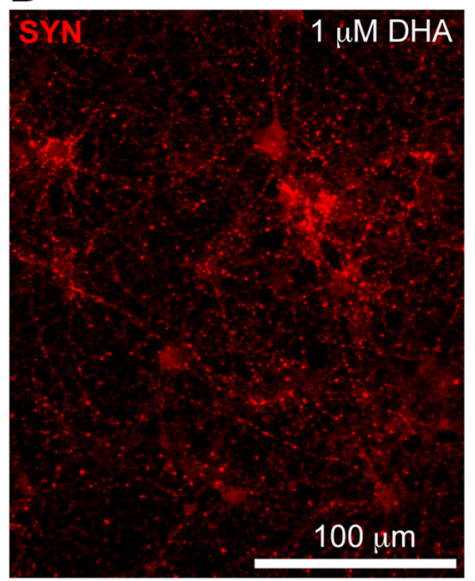

E
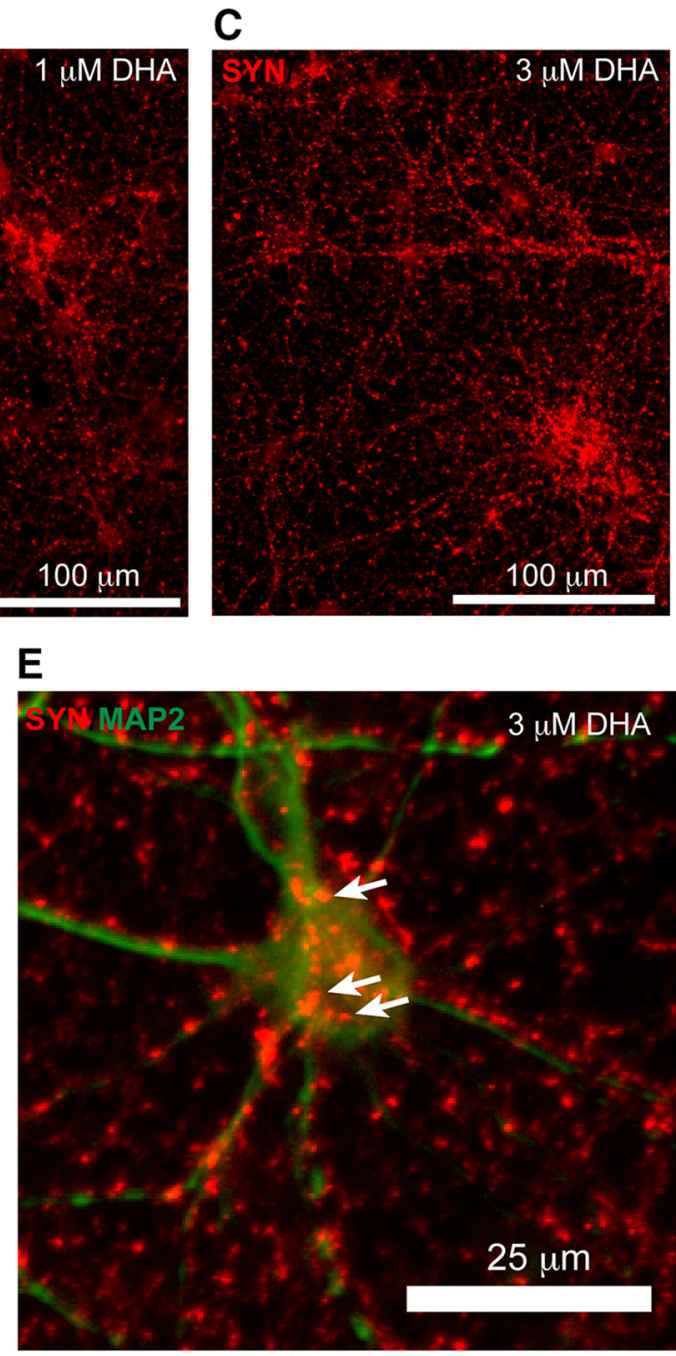

F

F Overall synaptophysin immunostaining

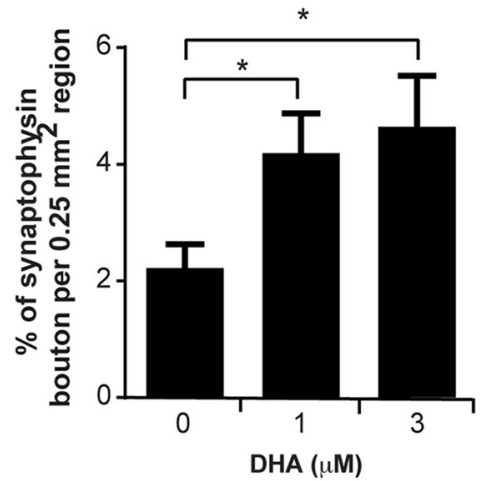

G
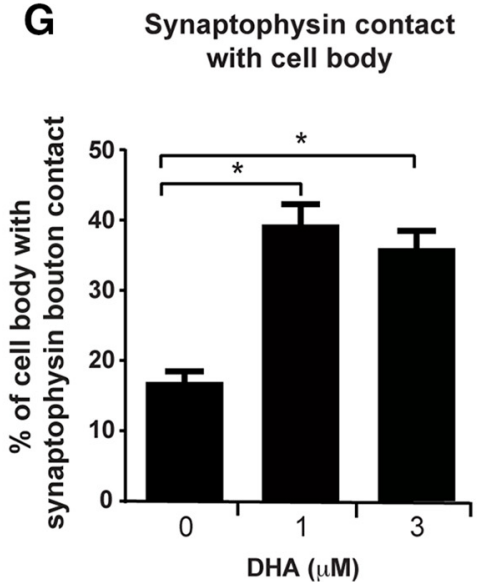

H

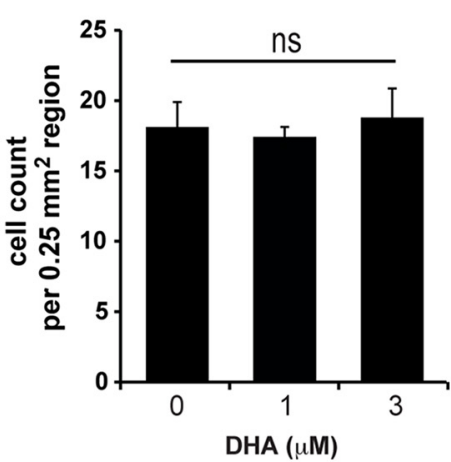

Figure 4. DHA enhances synaptophysin-immunopositive synaptic terminals in cortical neuron cell culture. $A-C$, Synaptophysin expression in embryonic cortical neuron cultures was assessed following a single application of a range of DHA concentrations (1-3 $\mu \mathrm{M}) . \boldsymbol{D}, \boldsymbol{E}$, High-magnification image showing the distribution of synaptophysin terminals (red) surrounding MAP2-immunopositive neuronal soma and neurites (green). $\boldsymbol{F}$, Global synaptophysin immunoreactivity in cortical neuronal cultures was significantly increased in both the 1 and $3 \mu \mathrm{m}$ DHA groups compared with the vehicle-treated group. $\mathbf{G}$, The density of synaptophysin contacts on cell bodies was increased with DHA treatment compared with vehicle treatment. $H$, No significant difference in the number of cortical neurons within a $0.25 \mathrm{~mm}^{2}$ area between treatment groups. $N=3$ or 4 independent experiments. ${ }^{*} p<0.05$, DHA versus vehicle group. ns, Not significant. Scale bars: $A-C, 100 \mu \mathrm{m} ; \mathbf{D}, \mathbf{E}, 25 \mu \mathrm{m}$.

comparable with the sham level (Fig. $5 J$ ). These data suggest that spontaneous serotonin fiber sprouting occurs in the absence of any treatment, but serotonin axonal sprouting is significantly enhanced in the presence of DHA after cervical lateral hemisection.
To gain further insight into the role of serotonin fibers in relation to motor function recovery, the density of serotoninimmunoreactive terminals contacting individual ventral horn motor neurons was analyzed (Fig. $5 K-M$ ). The analysis showed a significant increase in the density of serotonin-immunoreactive 

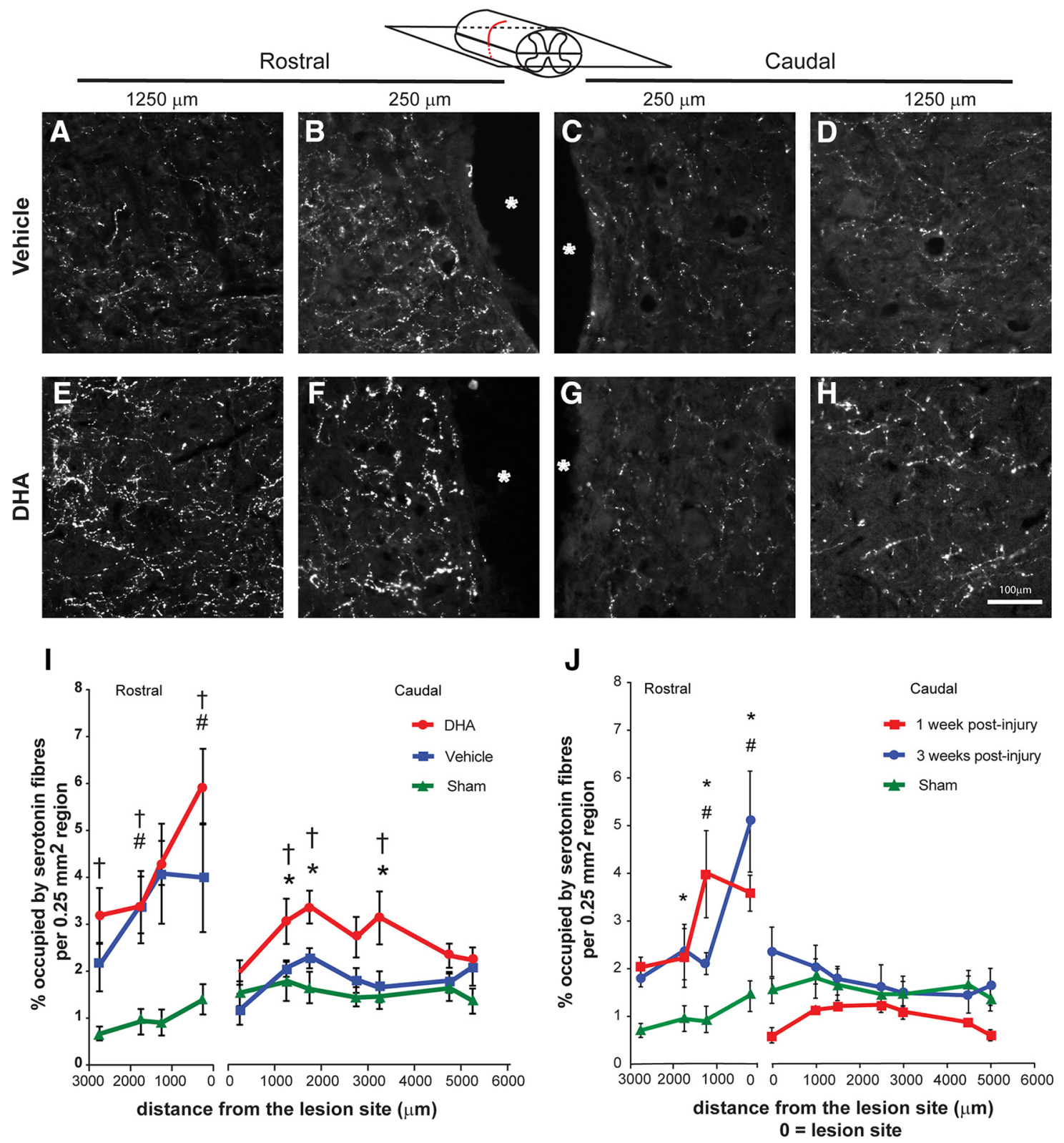

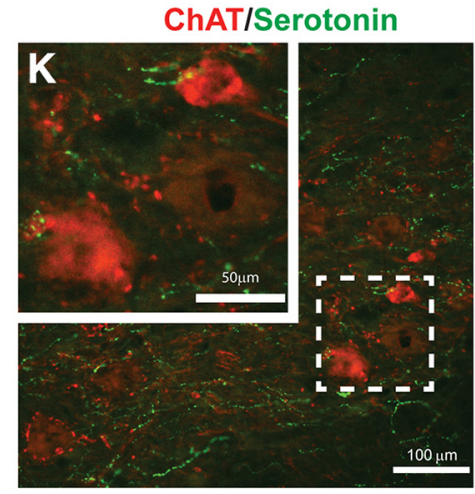

Veh
ChAT/Serotonin

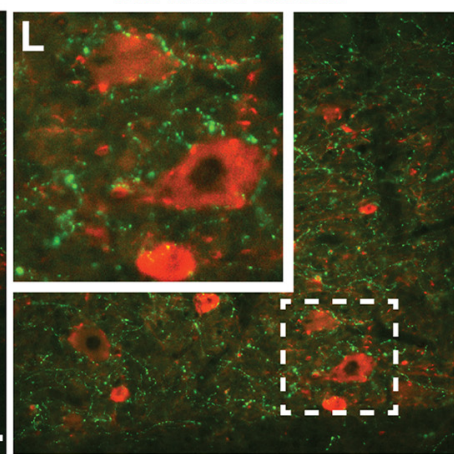

DHA

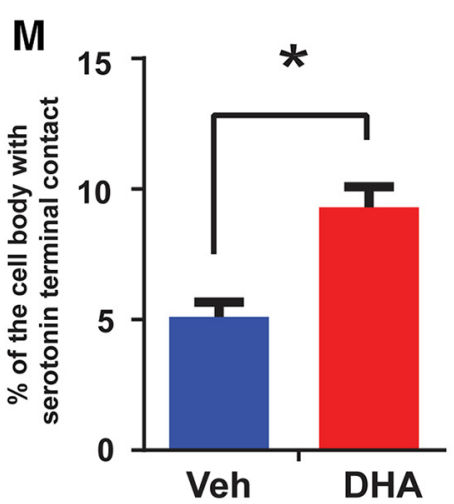

Figure 5. DHA enhances serotonin fiber regrowth following cervical lateral hemisection in the rat. $\boldsymbol{A}-\boldsymbol{H}$, Photomicrographs of serotonin-immunostained fibers in representative sections of the vehicle (top panels) and DHA-treated group (bottom panels) along the rostrocaudal axis of the hemisection lesioned side of the spinal cord in the horizontal plane. Stars indicate the lesion site. $I$, Serotonin fibers in the rostral part of the lesion site were significantly increased in both treatment groups, but only the DHA-treated group (red circles), not the vehicle group (blue squares), had significantly increased serotonin in the caudal region compared with the sham operated animals (green triangles).J, Serotonin fibers in the rostral part of the lesion site were significantly increased at 1 week (red square) and 3 weeks (blue circle) compared with the sham operated group after lateral cervical hemisection. However, in the caudal region, 1 and 3 weeks after injury were similar to the sham operated group. $N=5$ or 6 per group. ${ }^{\#} p<0.05,1$ week hemisection versus sham group. ${ }^{*} p<0.05,3$ week hemisection versus sham group. (Figure legend continues.) 
terminals contacting motor neurons in DHA-treated animals compared with the vehicle treatment (Fig. 5M). Therefore, overall, significant increases in the sprouting of serotonin fibers and the contacts with motor neurons were observed after cervical lateral hemisection and DHA treatment.

\section{Corticospinal projections}

To determine axonal sprouting in the voluntary motor system, CST axons caudal to the lesion site were analyzed. The tracer BDA was unilaterally injected into the ipsilateral hemisphere to anterogradely label uninjured CST axons and collaterals in rats following sham injury (Fig. 6A) and cervical lateral hemisection (Fig. $6 B, C$ ). In both the rostral and caudal regions on the injured side, there were significant increases in the number of BDAlabeled CST collaterals in the DHA-treated animals compared with the vehicle-treated group (Fig. 6D). Interestingly, DHAtreated animals had an increase in CST collateral sprouting on the unlesioned side at the rostral side compared with the vehicletreated animals (Fig. 6D). To confirm the BDA labeling efficiency of CST axons, the density of BDA-labeled CST at C1 level was analyzed (Fig. $6 F, G$ ). No significant difference was detected between the two treatment groups (Fig. $6 H$ ). These data suggest that DHA increased sprouting of corticospinal axons into the denervated cord region after a cervical lateral hemisection.

To further determine whether DHA can promote CST axon sprouting, a second animal species and injury model was used. Adult mice with a unilateral pyramidotomy were injected with a single bolus of DHA ( $500 \mathrm{nmol} / \mathrm{kg}$ ) or vehicle (saline with $0.2 \%$ ethanol) $30 \mathrm{~min}$ after injury. A higher dose of $500 \mathrm{nmol} / \mathrm{kg}$ was used in mice compared with $250 \mathrm{nmol} / \mathrm{kg}$ used in rats, based on our previous published data, showing species differences (Lim et al., 2013). Immunoreactivity for PKC $\gamma$ was used to confirm the unilateral lesion of one CST tract (Fig. $7 A-C$ ). A significant increase in the number of sprouting CST fibers was detected in the DHA-treated animals compared with the vehicle group (Fig. 7D$F$ ). However, sprouting CST fibers were not significantly detected in sham animals treated with DHA (Fig. $7 F$ ). In addition to the histological determination of increased sprouting of CST fibers, behavioral analysis was performed to determine whether functional recovery was observed with a single bolus injection of DHA. During the 4 week period after CST injury, the grid exploration test was used to assess sensorimotor function in DHA and vehicle-treated mice. No significant difference was detected between the groups in forelimb grid exploration in the first 2 weeks (Fig. 8A). However, 3 weeks after injury, DHA-treated mice had significantly fewer misplacements compared with vehicle treatment (Fig. 8A). In the grid exploration test involving the hindlimb, fewer misplacements were observed in the DHAtreated group compared with the vehicle group, from 2 weeks after injury, but the difference was not statistically significant (Fig. 8B).

Interestingly, the significant increase in CST fibers on the CST-denervated side occurred in the intermediate laminae and

\footnotetext{
$\leftarrow$

(Figure legend continued.) $\quad \boldsymbol{K}, \boldsymbol{L}$, The represented images are captured $1750 \mu \mathrm{m}$ caudal to lesion site. Double labeling shows serotonin fibers (green) in contact with ChATimmunopositive motor neurons (red) in the ventral horn of vehicle- and DHA-treated rats. Insets, Dashed boxes at higher magnification. $\boldsymbol{M}$, Quantitative analysis reveals that there is a significant increase in the density of serotonin fibers contacting motor neurons in the DHAtreated group compared with the control group. $N=5$ or 6 per group. ${ }^{*} p<0.05$, DHA versus vehicle group. $t p<0.05$, sham versus vehicle group. ${ }^{*} p<0.05$, DHA versus sham group. Scale bar, $100 \mu \mathrm{m}$.
}

not in the ventral horn near motor neurons, which suggests that the improved functional outcome involved interneurons. One potential type of interneuron is the V2a interneuron, which is responsible for locomotion control and coordination in mice (Al-Mosawie et al., 2007; Dougherty and Kiehn, 2010) and more precise skilled forelimb movement (Azim et al., 2014). Using immunohistochemistry, V2a interneurons in the spinal cord could be identified using a Chx10 antibody (Dougherty and Kiehn, 2010). Double immunolabeling revealed that sprouting CST fibers appeared to contact V2a interneurons. Confocal analysis of selected sections confirmed that some CST fibers made contacts with Chx10-immunoreactive cell bodies, revealed using blue fluorescent Nissl staining (Fig. 8C-F). Furthermore, CST fibers contacted significantly more $\mathrm{V} 2 \mathrm{a}$ interneurons in the DHA-treated group than in the vehicle group (Fig. $8 G$ ). The analysis revealed a negative correlation between the number of Chx10-immunopositive V2a interneurons contacted by CST fibers and the number of forelimb misplacements observed on the grid exploration test (Fig. $8 H$ ). These data suggest that DHA induces uninjured CST collaterals to sprout across the midline and contact V2a interneurons, ultimately leading to improved functionality proximal to the injury region.

\section{DHA can suppress the levels of the inhibitory regenerative molecule PTEN}

Studies have recently shown that the PTEN molecule, which inhibits regeneration, peaks at $1 \mathrm{~d}$ and then decreases at $3 \mathrm{~d}$ after injury, in various rat CNS injury models (Ding et al., 2013; Hu et al., 2013). We therefore repeated the cervical lateral hemisection experiment and perfused the animals at $1 \mathrm{~d}$ after injury to capture the acute effect of DHA on PTEN levels. High PTEN levels were observed, colocalizing with NeuN-positive neurons in the primary motor cortex of hemisected animals at $1 \mathrm{~d}$ after injury (Fig. $9 A-F)$. However, DHA-treated animals showed a significantly reduced PTEN expression in layer $\mathrm{V}$ cortical neurons compared with the vehicle group (Fig. $9 A-F, K$ ). The PTEN expression of sham operated animals was similar to naive animals (Fig. $9 G-J$ ).

In adult primary cortical neuron cultures, $3 \mu \mathrm{M}$ DHA significantly reduced the basal PTEN levels (Figs. 10A,B,E, $11 A, B$ ). DHA-treated cultures, at 1 and $3 \mu \mathrm{M}$, exhibited significantly greater maximum neurite length compared with the control group (Fig. 10A,B,E,F). This suggests that DHA can reduce PTEN, and this effect could underlie increased axonal growth and the modulation of neuroplasticity.

\section{DHA can alter the AKT/mTOR pathway}

Recently, DHA has been shown to be a positive modulator of AKT signaling in neuronal survival (Akbar et al., 2005). We therefore wanted to investigate whether DHA can upregulate phosphorylated AKT (pAKT) in primary cortical neurons. $\mathrm{DHA}$ at 1 and $3 \mu \mathrm{M}$ significantly increased the expression of pAKT compared with the control group (Figs. $10 C, D, H$, $11 C, D)$. Furthermore, as mTOR is downstream of the PI3KAKT pathway, inhibition of the mTOR should inhibit the effect of DHA. In the presence of rapamycin (30 nM), an mTOR inhibitor, no significant effect on the maximum neurite length was observed with 1-3 $\mu \mathrm{M}$ DHA (Fig. 10H). This suggests that DHA can increase axonal growth and modulate neuroplasticity via the AKT-mTOR pathway.

\section{DHA can upregulate the expression of the miR-21}

The microRNA miR-21 is one of the most prominent miRNAs expressed following SCI (Hu et al., 2013; Nieto-Diaz et al., 2014). 


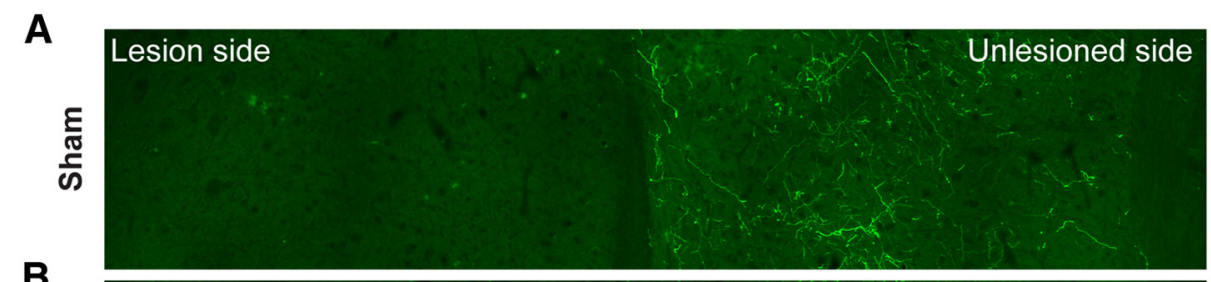

B
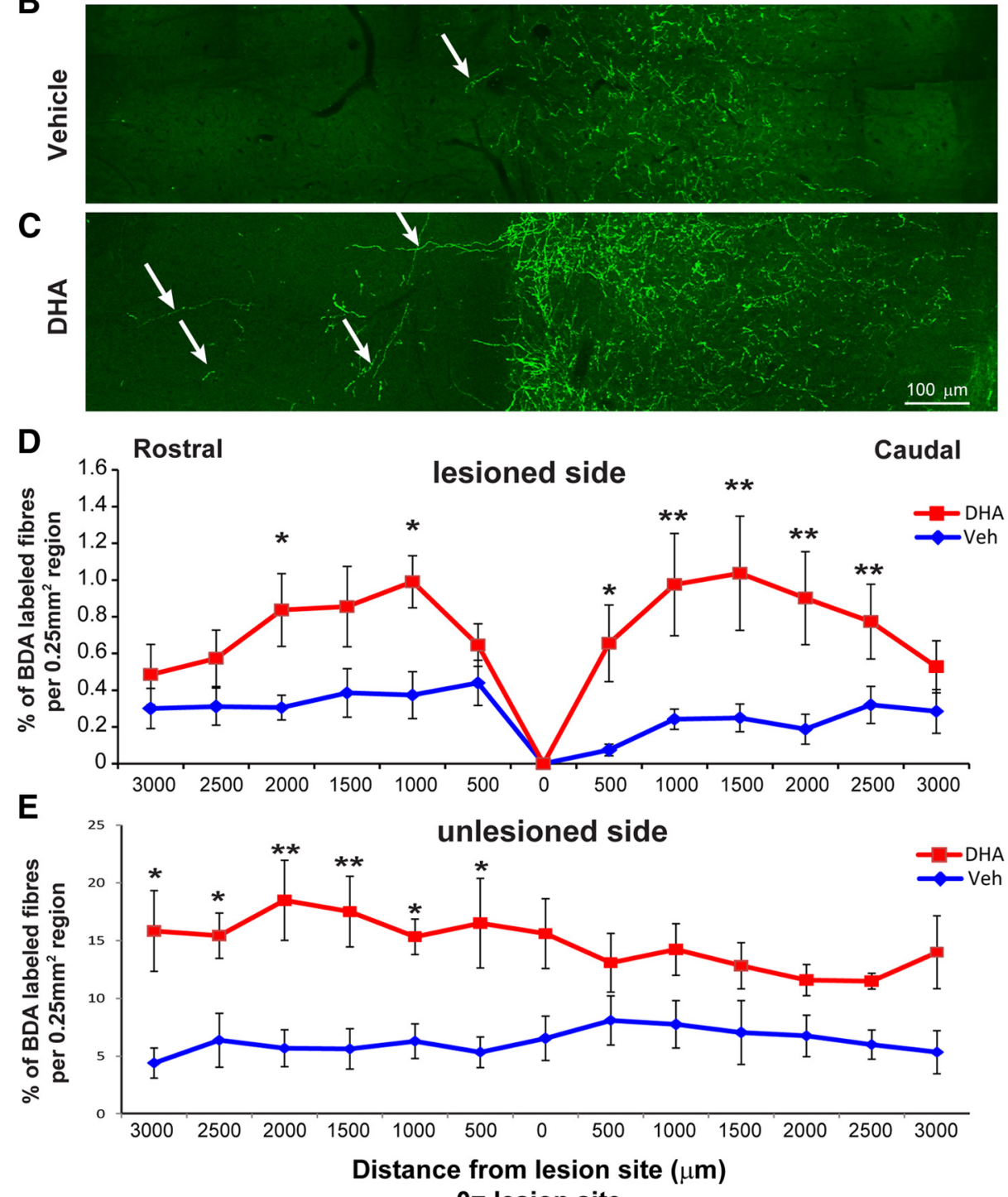

$\mathbf{F}$

G

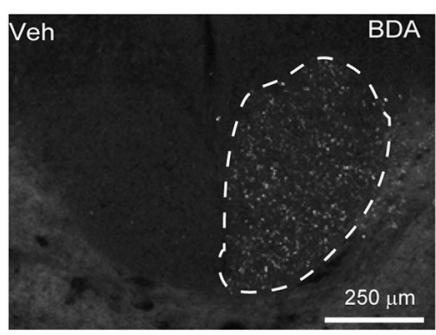
$0=$ lesion site

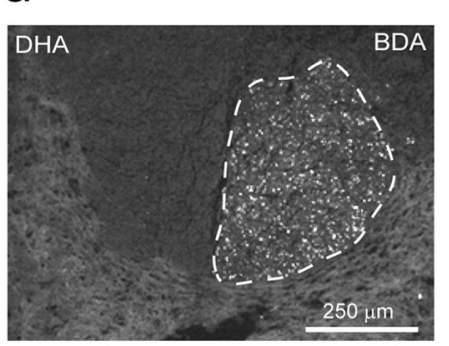

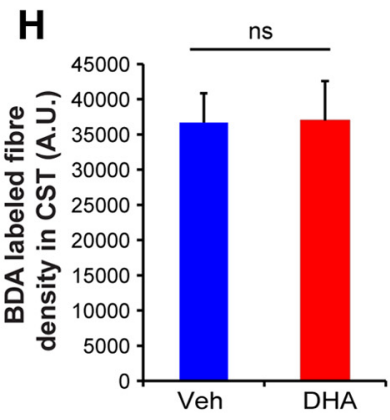

Figure 6. DHA enhances sprouting of corticospinal axons following cervical lateral hemisection in rats. $A-C$, Photomicrographs of horizontal sections $1000 \mu \mathrm{m}$ caudal to the lesion site reveal more sprouting of BDA-labeled CST collaterals (arrows) across the midline into the lesion side in the DHA-treated group compared with the vehicle-treated and sham operation group. $\boldsymbol{D}, \boldsymbol{E}$, Quantitative analysis showed a significant increase in BDA-labeled CST fibers at certain levels of the spinal cord rostral and caudal to the injury on both the lesioned and unlesioned side in DHA-treated animals (red circle) compared with the vehicle-treated animals (blue square). ${ }^{*} p<0.05$, DHA versus the vehicle group. ${ }^{* *} p<0.01$, DHA versus the vehicle group. Scale bar, $100 \mu \mathrm{m}$. $\boldsymbol{F}-\boldsymbol{H}$, No significant difference was found in the number of BDA-labeled CST axons at the C1 dorsal funiculus between the DHA and vehicle-treated groups. ns, Not significant. 


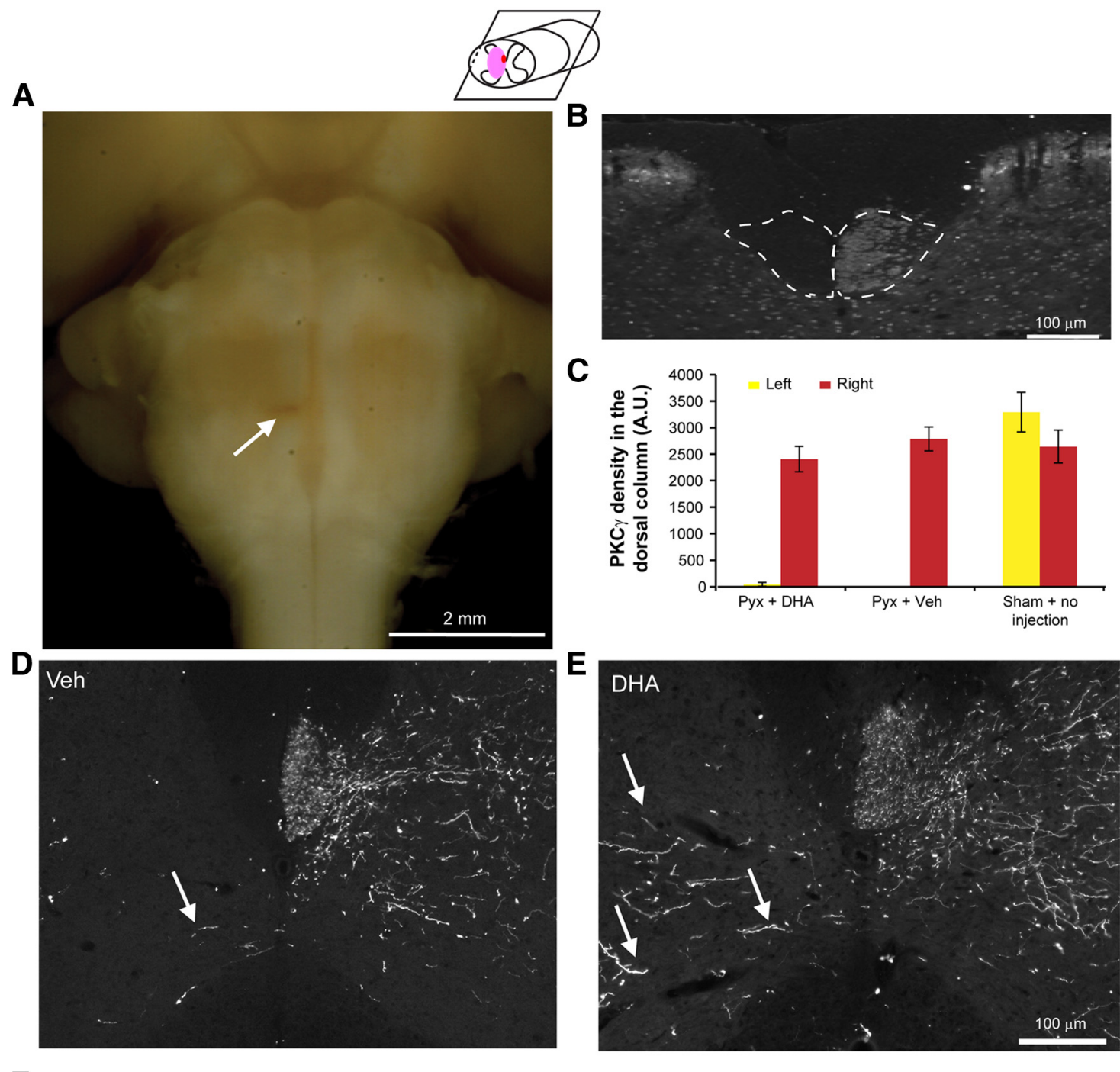

$\mathbf{F}$

CST-denervated side
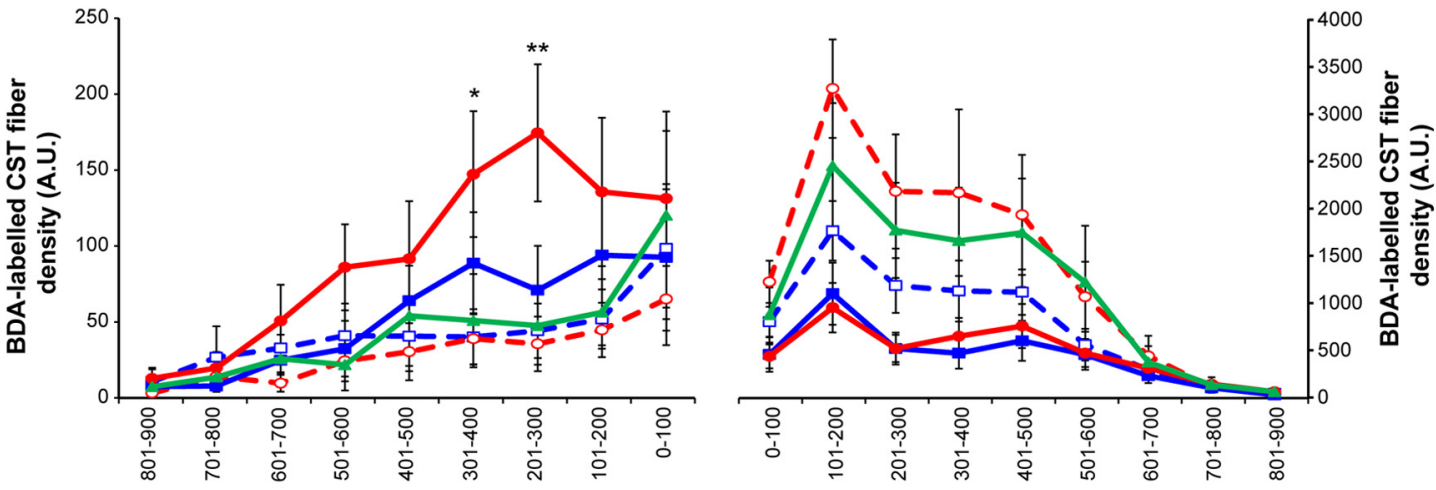

Distance from midline $(\mu \mathrm{m})$

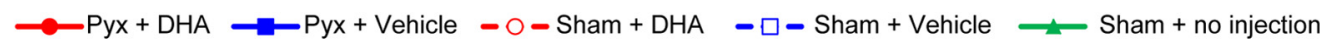

Figure 7. DHA enhances corticospinal axon sprouting following pyramidotomy in mice. $A$, Ventral view of a mouse brainstem with a unilateral pyramidotomy. Arrow indicates a unilateral lesion of the CST located in the medullary pyramids. $B$, Lack of PKC $\gamma$ immunostaining in the left ventral portion of the dorsal funiculus of the cervical spinal cord confirms the unilateral lesion of the CST. C, The intensity of PKC $\gamma$ immunostaining of the lesioned CST in the cervical spinal cord reveals a significant decrease after pyramidotomy compared with the uninjured CST tract and the sham operated group. $\boldsymbol{D}, \boldsymbol{E}$, Transverse sections of the cervical spinal cord reveal CST fibers sprouting from the contralateral side across the midline after pyramidotomy in both vehicle and DHA groups. $\boldsymbol{F}$, Distance-specific analysis of midline crossing fibers shows that the DHA-treated group (red circles) had significantly more midline crossing fibers compared with vehicle (blue squares) and the sham operated group (green triangles) on the (ST-denervated side of the cervical spinal cord. Sham operated animals, which received either DHA (red open circles) or vehicle (blue open squares), did not show a significant difference compared with the sham operated group. ${ }^{*} p<0.05$, versus the vehicle group. ${ }^{* *} p<0.01$, DHA versus the vehicle group. 

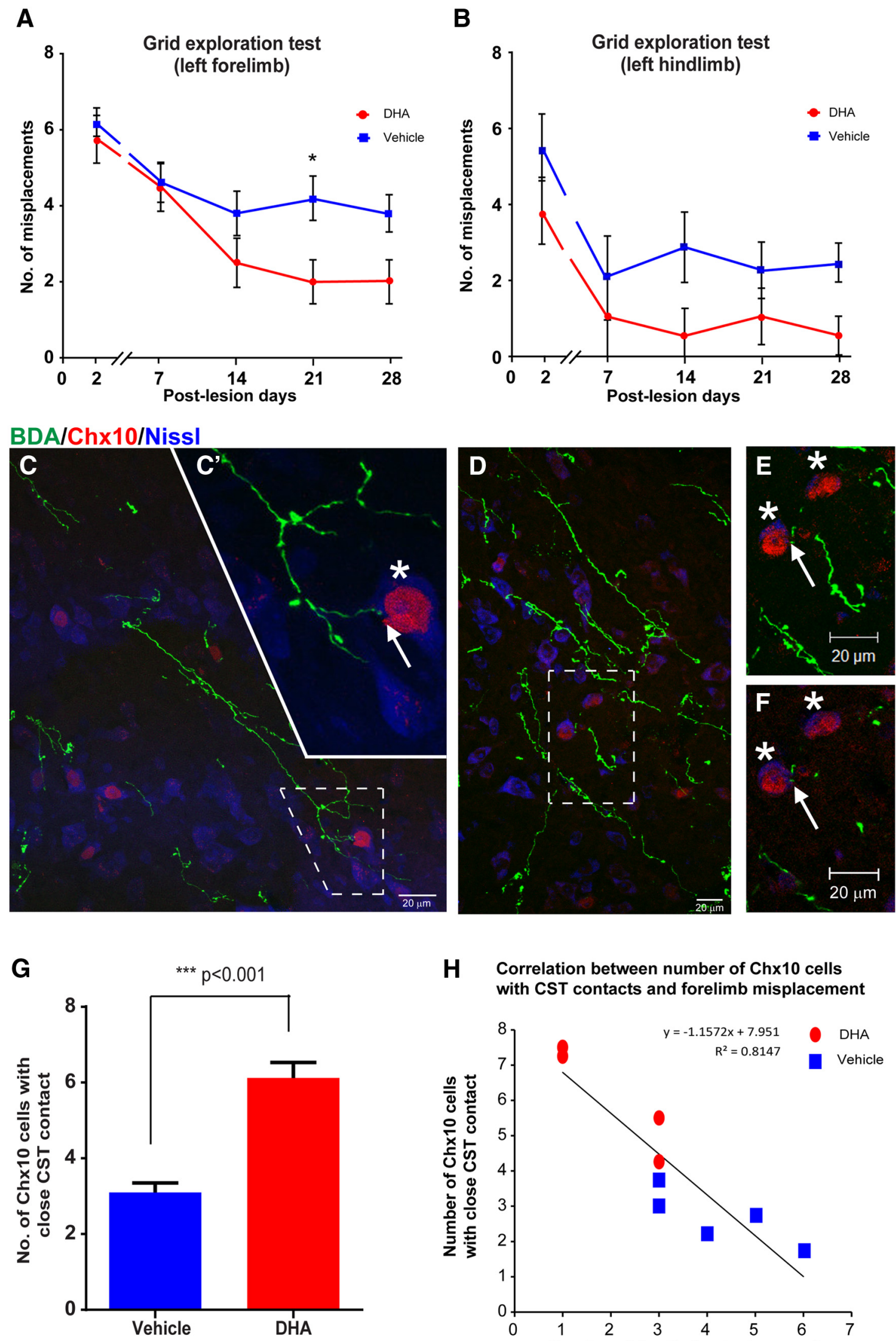

H Correlation between number of Chx10 cells with CST contacts and forelimb misplacement

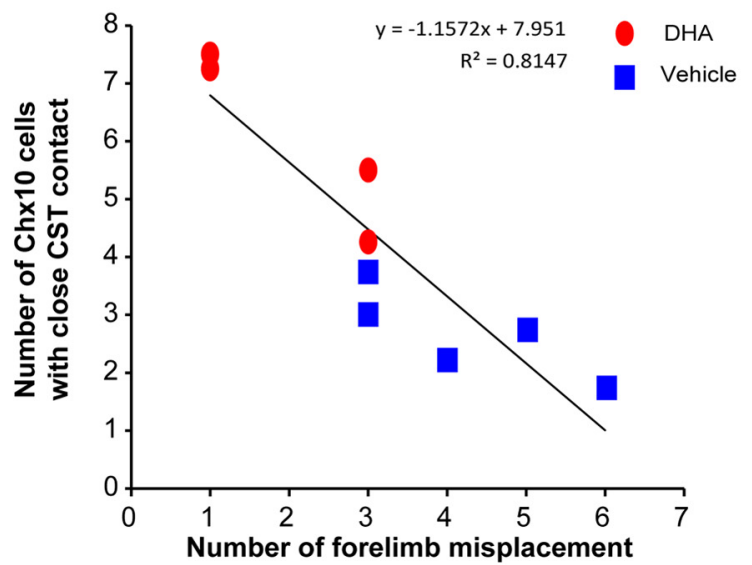

Figure 8. DHA enhances functional recovery via connection with Chx10 interneurons in pyramidotomy mice. $A$, Mice with a unilateral pyramidotomy failed to initially accurately place the left forepaw (requiring integrity of the lesioned CST) onto the grid after injury, but the DHA-treated rats (red circles) made significantly fewer misplacements at 3 weeks after injury compared with vehicle-treated mice (blue squares). $\boldsymbol{B}$, The analysis revealed that DHA-treated mice (red circles) made fewer errors with their left hindpaw, but not significantly less than vehicle group animals (blue squares) throughout the testing period. $\mathbf{C}-\boldsymbol{F}$, Confocal images of mouse cervical spinal cord transverse sections at the intermediate laminae of the CST-denervated (Figure legend continues.) 

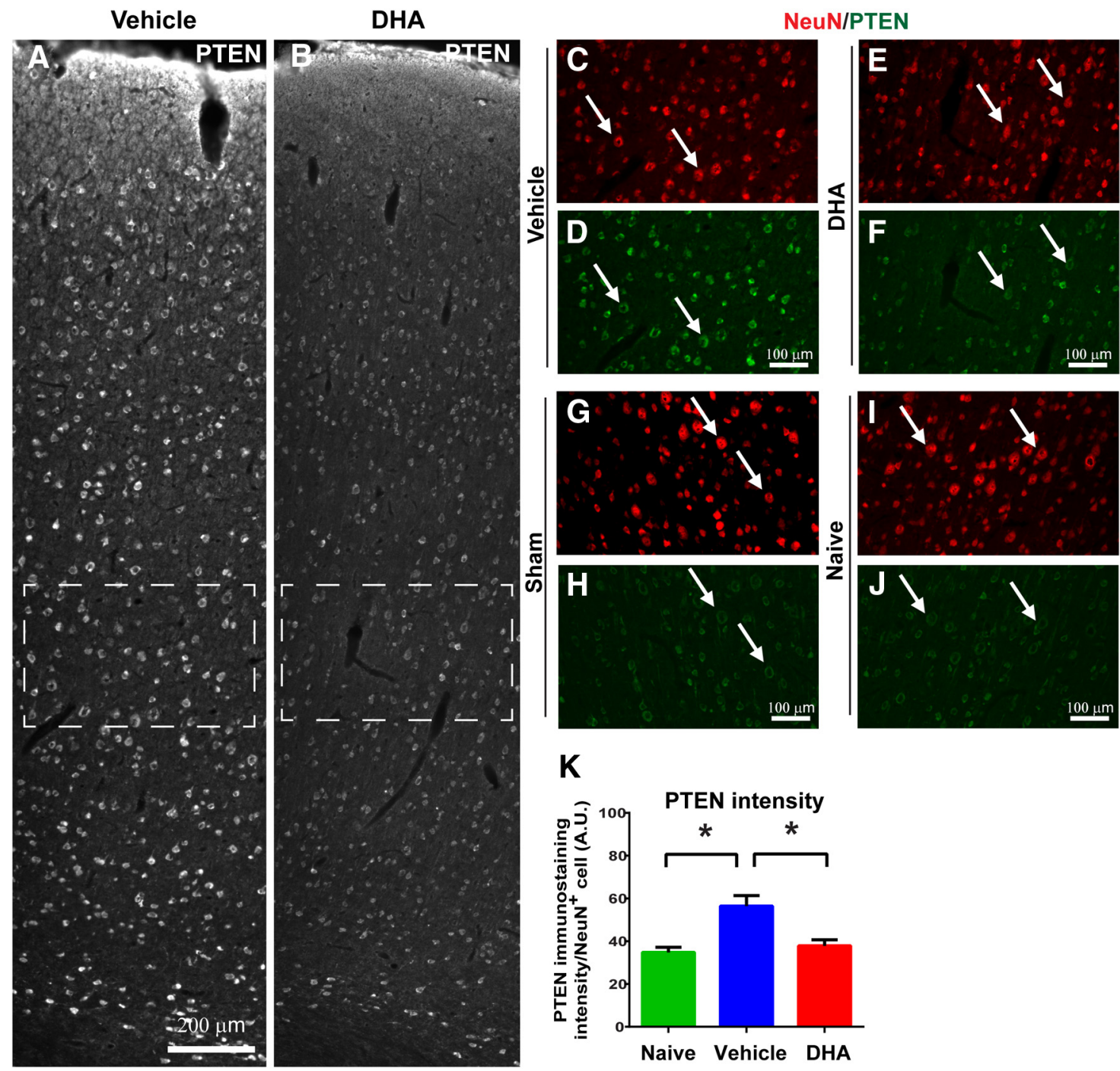

Figure 9. DHA reduces intracellular PTEN levels in cortex after cervical hemisection. $A, B$, Low-magnification images of the cerebral cortex labeled for PTEN immunoreactivity in cervical lateral hemisected rats at $1 \mathrm{~d}$ after injury. $\mathbf{C}-\boldsymbol{F}$, Higher magnification of the dashed boxes in $\boldsymbol{A}, \boldsymbol{B}$ showing that PTEN immunoreactivity is colocalized with NeuN-immunopositive neurons in the cerebral cortex. DHA-treated animals have reduced PTEN immunostaining compared with vehicle-treated animals. $G, J$, A low expression of PTEN immunoreactivity was observed in the cortex of naive and sham animals. $\boldsymbol{K}$, Quantitative analysis revealed a significant increase in PTEN immunostaining in the cerebral cortex ipsilateral to the lesion side at $1 \mathrm{~d}$ after cervical SCl in the vehicle group. DHA treatment (red bar) significantly reduces the PTEN immunostaining levels compared with the vehicle treatment (blue bar). ${ }^{*} p<0.05$. Scale bars: $A, B, 200 \mu \mathrm{m} ; C-J, 100 \mu \mathrm{m}$.

Manipulation of the level of miR-21 has been shown to affect functional recovery by modulating PTEN expression in CNS injury animal models (Hu et al., 2013; Ge et al., 2014). Here, we used ISH to examine the level of miR-21 in neurons in the pri-

\footnotetext{
(Figure legend continued.) side showing examples of BDA-labeled CST collaterals (green) in the vicinity of blue fluorescent Niss--stained cells (Neurotrace 435/455), some of which are V2a interneurons identified by immunostaining for $\mathrm{Chx} 10$ (red). C, D, Dashed boxes represent high magnification in $\boldsymbol{C}^{\prime}$, and in $\boldsymbol{E}, \boldsymbol{F}$, which reveal contacts (arrows) between BDA-labeled collaterals and Chx10 interneurons (asterisks). $\boldsymbol{C}^{\prime}, \mathrm{A} Z$-stack comprising $10 \times 0.66 \mu \mathrm{m}$ optical images. $\boldsymbol{E}$, AZ-stack comprising $25 \times 0.72 \mu \mathrm{m}$ optical images. $\boldsymbol{F}$, A single $0.72 \mu \mathrm{m}$ optical image. Examination of the single optical image confirms that the BDA-labeled CST fiber contacts (arrow) one of the Chx10 interneurons. $G$, Quantitative analysis revealed a significant increase in the number of Chx10 interneurons contacted by BDA-labeled CST collaterals following DHA treatment (red bar) compared with vehicle treatment (blue bar). $\boldsymbol{H}$, A strong negative correlation was observed between the numbers of $\mathrm{Chx} 10$ interneurons with BDA-labeled CST contacts and the numbers of forelimb misplacements. Data were taken from DHAtreated (red circle) and vehicle-treated (blue square) animals. ${ }^{* *} p<0.001$, DHA versus vehicle group. Scale bar, $20 \mu \mathrm{m}$.
}

mary motor cortex at $1 \mathrm{~d}$ after injury. After DHA treatment, the level of miR-21 expression in layer $\mathrm{V}$ cortical neurons was significantly upregulated compared with the vehicle group (Fig. 12A$F)$. In cortical neuron cultures, miR-21 was also shown to be upregulated after DHA treatment, using qRT-PCR (Fig. 12G). Furthermore, in the presence of a miR-21 inhibitor $(3 \mathrm{pmol} / \mathrm{ml})$, DHA could not significantly decrease PTEN expression; and there was no increase in average neurite length (Fig. 10I,J). On the contrary, the average neurite length was significantly reduced in the presence of 0 and $1 \mu \mathrm{M}$ DHA. This suggests that the proregenerative effect of DHA involves an upregulation of miR-21.

\section{Discussion}

Several recent studies have shown that DHA promotes beneficial functional effects in SCI (King et al., 2006; Huang et al., 2007; Figueroa et al., 2013; Lim et al., 2013), traumatic brain injury (Wu et al., 2004; Bailes and Mills, 2010; Russell et al., 2013; Begum et al., 2014; Desai et al., 2014), ischemic injury (Pan et al., 2009; Eady et al., 2012), and Alzheimer's disease (Hashimoto et 
A

PTEN/ $\beta 3$ tubulin
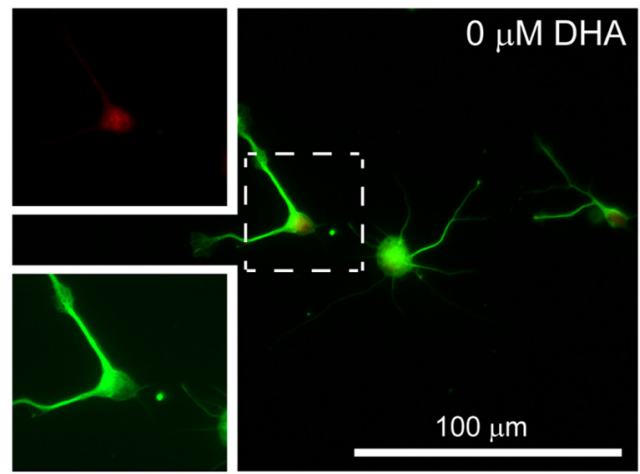

C

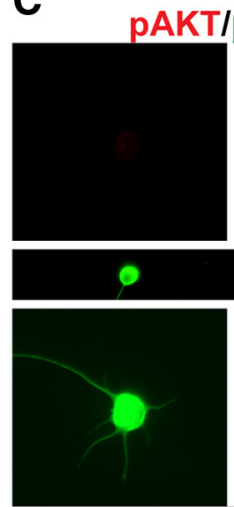

E

PTEN expression

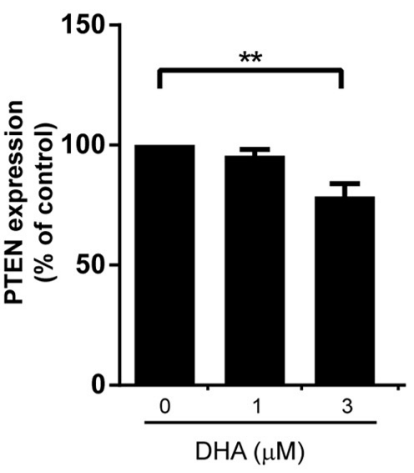

H Maximum neurite length I with rapamycin

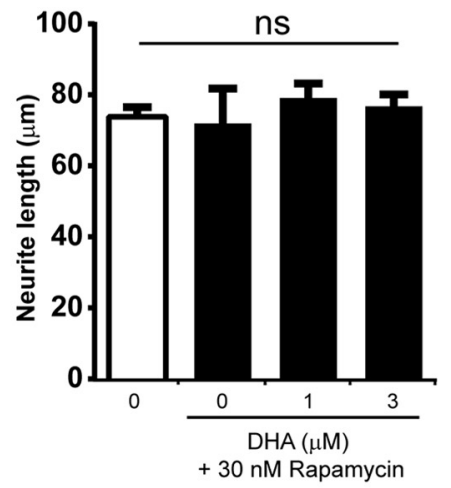

B

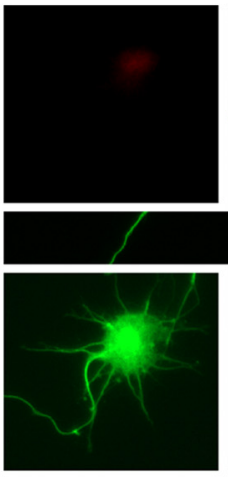

D

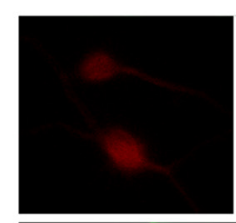

pAKT/ 33 tubulin

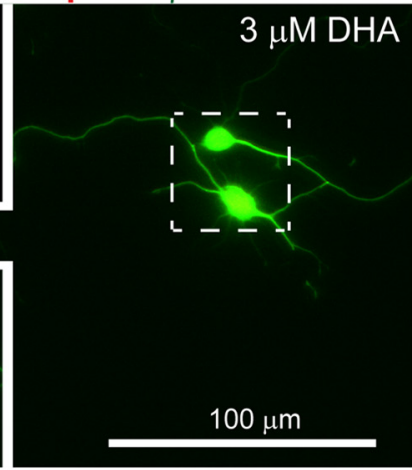

G pAKT expression

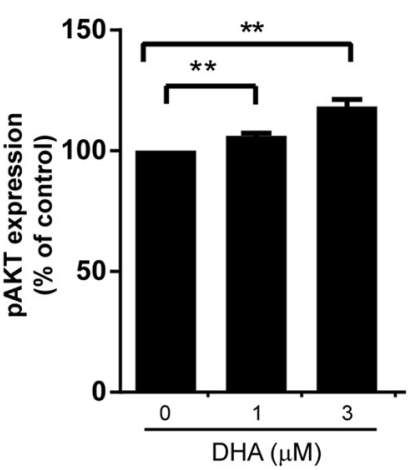

J

PTEN expression with miR-21 inhibitor

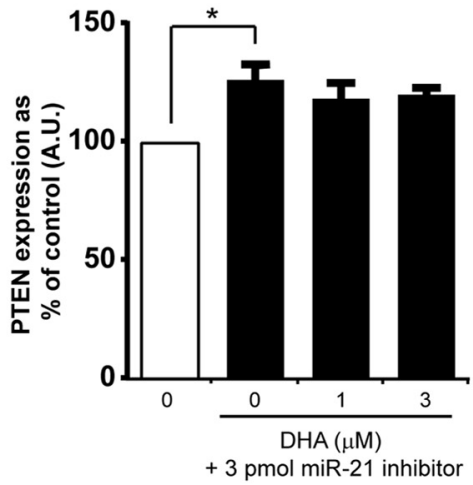

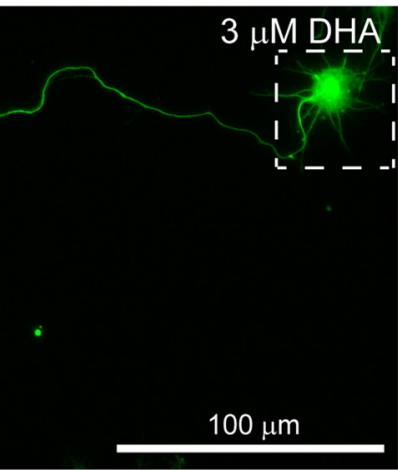

PTEN/ $\beta 3$ tubulin

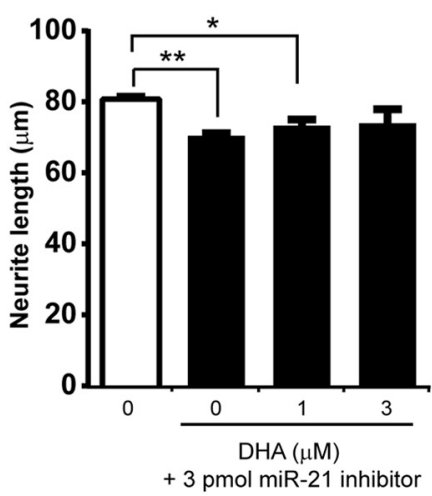

Figure 10. DHA promotes neurite growth through the PTEN/pAKT/mTOR signaling pathway by miR-21 modulation. $A-G$, In embryonic cortical neuron cultures, $3 \mu \mathrm{m}$ DHA significantly enhances the neurite growth with concomitant reduction in PTEN and increase in PAKT level. $\boldsymbol{H}$, After rapamycin treatment, no significant neurite outgrowth was observed after incubation with DHA. $I, J, I n$ cortical neurons transfected with miR-21 inhibitor, the ability to suppress PTEN expression and promote neurite growth by DHA was attenuated. ${ }^{*} p<0.05$, ${ }^{* *} p<0.01$. ns, Not significant. 

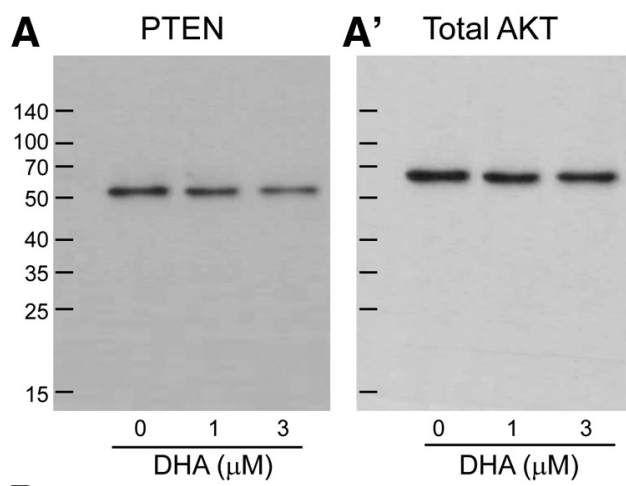

B
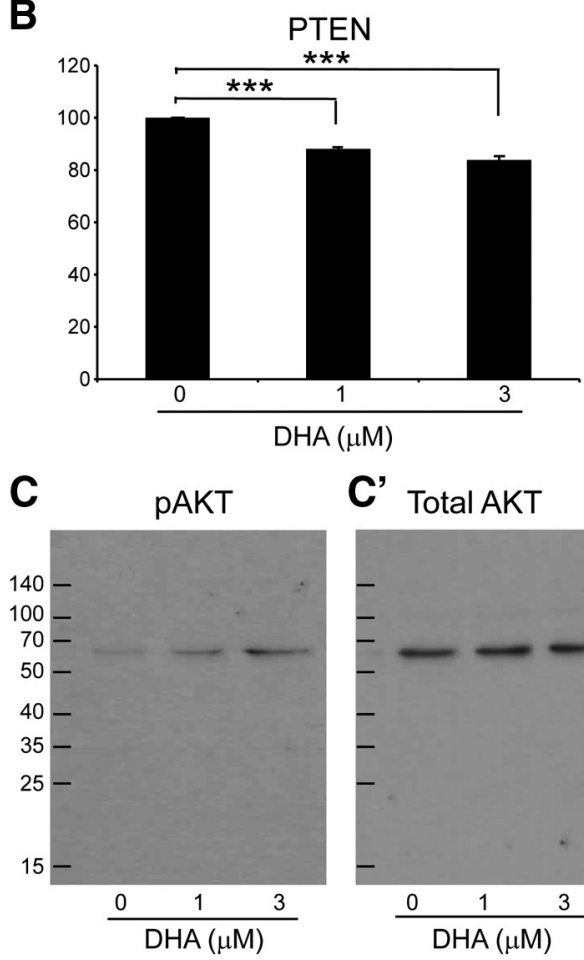

C' Total AKT

D

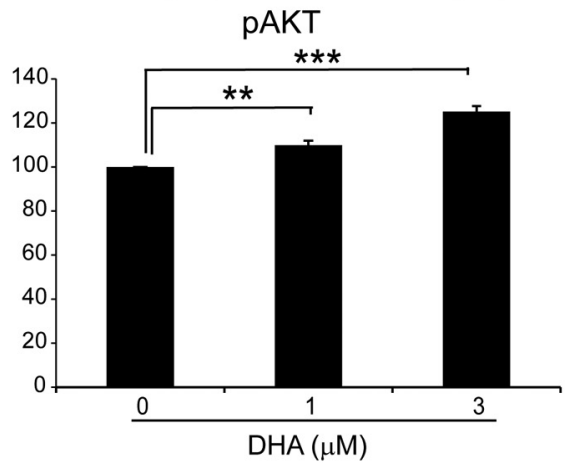

Figure 11. DHA downregulates PTEN expression and upregulates p-AKT expression in vitro. The expression levels of PTEN and PAKT in cortical neurons were measured by Western blot. The qualitative expression of PTEN and pAKT is shown $(\boldsymbol{B}, \boldsymbol{D}) . \boldsymbol{A}, \boldsymbol{B}$, Quantitative analysis of PTEN protein levels revealed a significant decrease after DHA treatment. $\boldsymbol{C}, \boldsymbol{D}$, Quantitative analysis of pAKT protein levels revealed a significant increase after DHA treatment. $A^{\prime}, C^{\prime}$, Blots of total AKT after stripping the corresponding membrane with Thermo Scientific restore plus Western blot stripping buffer. Gray value analysis of each band was performed, and their ratios in correspondence to the total AKT band were calculated. The results demonstrated that 1 and $3 \mu \mathrm{m}$ DHA significantly increase PTEN and decrease pAKT expression in cortical neurons ${ }^{* *} p<0.001 .{ }^{* *} p<0.001 . N=3$ or 4 independent samples. al., 2002; Lim et al., 2005). However, the mechanisms underlying these functional changes remain poorly understood. This present study demonstrates that a single intravenous bolus of DHA can induce functional neuroplasticity by promoting anatomical sprouting, in two different rodent models of SCI. Anatomical plasticity was demonstrated by the fact that DHA caused an increase in serotonin and BDA- immunolabeled CST axons caudal to a hemisection injury. Furthermore, an increase in the number of synaptic boutons was demonstrated both in vivo and in vitro following treatment with DHA. In the hemisection and the compression injury model, DHA is neuroprotective (Huang et al., 2007), and it is therefore possible that the behavioral improvement reported was due to neuroprotection of the cord rather than neuroplasticity. However, in a pyramidotomy injury, in which there is no direct injury to the spinal cord, DHA-induced CST sprouting was also observed, confirming that DHA promotes neuroplasticity. CST axons contacted V2a interneurons, which are responsible for locomotion control and coordination (Al-Mosawie et al., 2007; Dougherty and Kiehn, 2010; Azim et al., 2014), and these new contacts likely contributed to the behavioral recovery (see below).

It is remarkable that a single injection of DHA administered $30 \mathrm{~min}$ after injury causes long-lasting changes. However, there have been several studies that have shown effective functional and histological improvements using a similar paradigm (King et al., 2006; Huang et al., 2007; Belayev et al., 2009, 2011; Ward et al., 2010; Lim et al., 2013; Paterniti et al., 2014). Interestingly, when a single bolus DHA treatment was delayed for 3 weeks or even $3 \mathrm{~h}$ after SCI, the functional improvement of DHA was diminished in the rat hemisection (Fig. 2) and compression models (Huang et al., 2007), and this is similar to the time dependence reported in stroke models (Belayev et al., 2011). These data suggest that the mechanism of DHA involves a critical step at an early time point. One potential molecular target is PTEN, a central negative regulator of the phosphatidylinositol 3-kinase (PI3K) signaling pathway. This protein, which is present in most central neurons, has been reported to be upregulated following SCI, with a peak at $1 \mathrm{~d}$ after traumatic injury and then reduced at $3 \mathrm{~d}$ (Ding et al., 2013; $\mathrm{Hu}$ et al., 2013). PTEN has been shown to be involved in axonal regeneration in optic nerve (Park et al., 2008), corticospinal neurons (Liu et al., 2010), sensory neurons (Christie et al., 2010), and synaptic plasticity (Liu et al., 2012). PTEN is also an important target of miR-21. Some studies report that upregulation of miR-21 can improve functional recovery by suppressing PTEN (Han et al., 2014; Sandhir et al., 2014). Our study showed that DHA significantly increases miR-21 level in cortical neurons in vivo and reduces PTEN expression in neurons both in vivo and in $v i t r o$, and this critical modulation occurs early after injury. Three weeks after injury, PTEN was still reduced in DHA-treated animals, but this difference from vehicle-treated animals was no longer significant (data not shown). DHA downregulated intracellular PTEN throughout the cortex, with a significant decrease observed in pyramidal neurons in layer $\mathrm{V}$ of the primary motor cortex. In cortical neuron cultures, DHA promoted significantly greater neurite length and a concomitant reduction in PTEN and an increase in pAKT shown using immunostaining and Western blotting. Furthermore, in the presence of either the mTOR inhibitor rapamycin, or a miR-21 inhibitor, the increase in neurite growth induced by DHA was attenuated. These data suggest that DHA has an effect on the PTEN-AKT-mTOR pathway, with involvement of miR-21. The effect of DHA on this pathway is in agreement with Akbar et al. (2005), showing DHA as a positive modulator of AKT signaling in Neuro-2A cells, and the evidence 


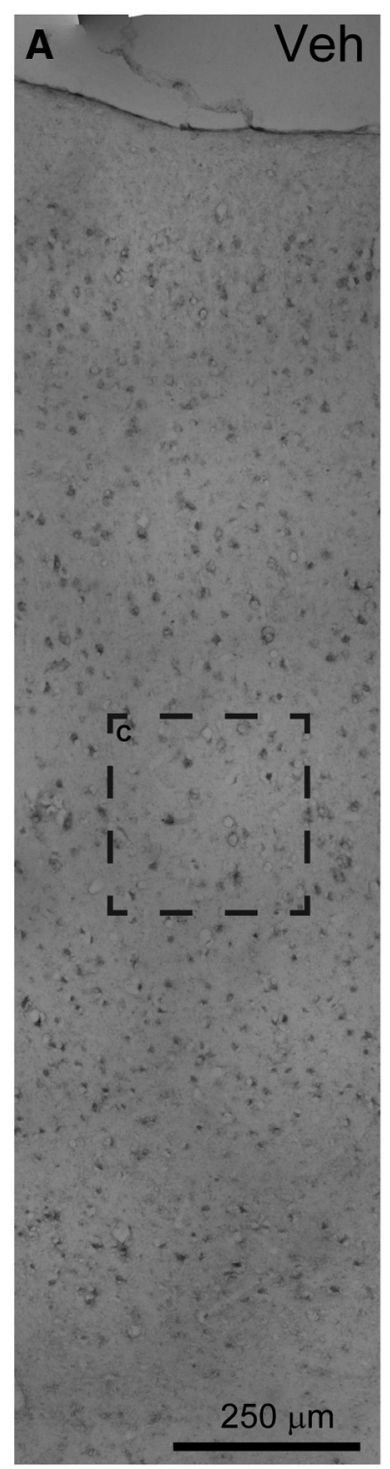

$\mathbf{F}$
In vivo miR-21 expression in layer V cortical neurons

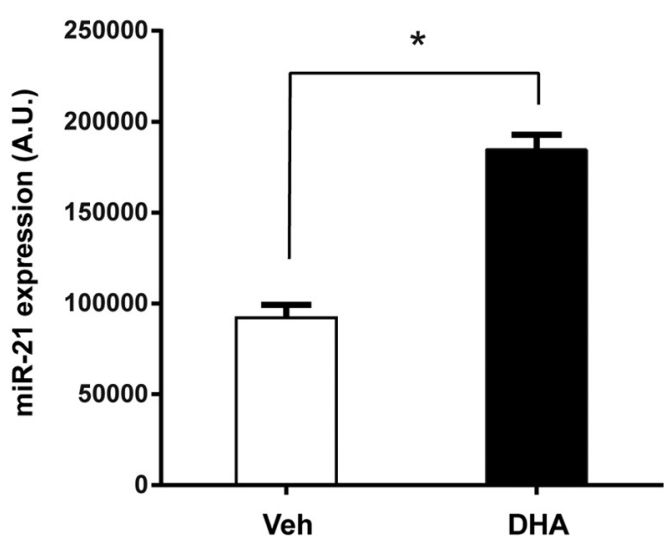

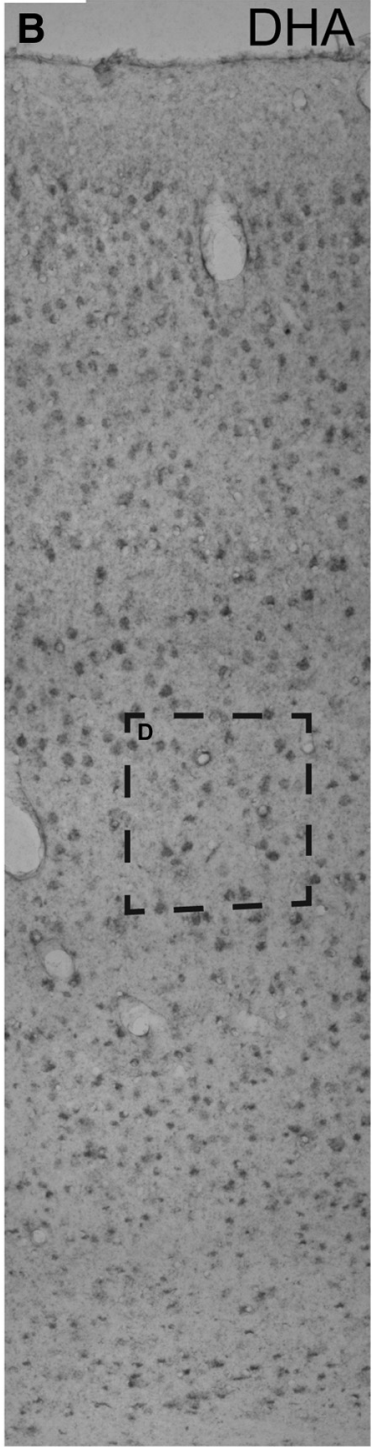
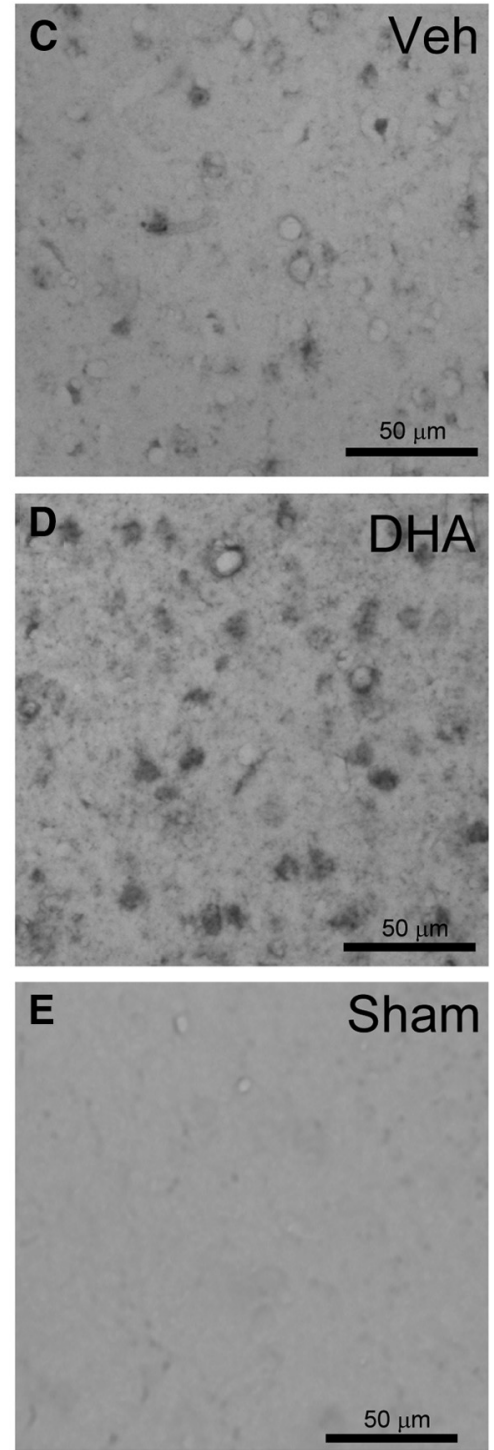

G In vitro miR-21 expression in cultured cortical neurons

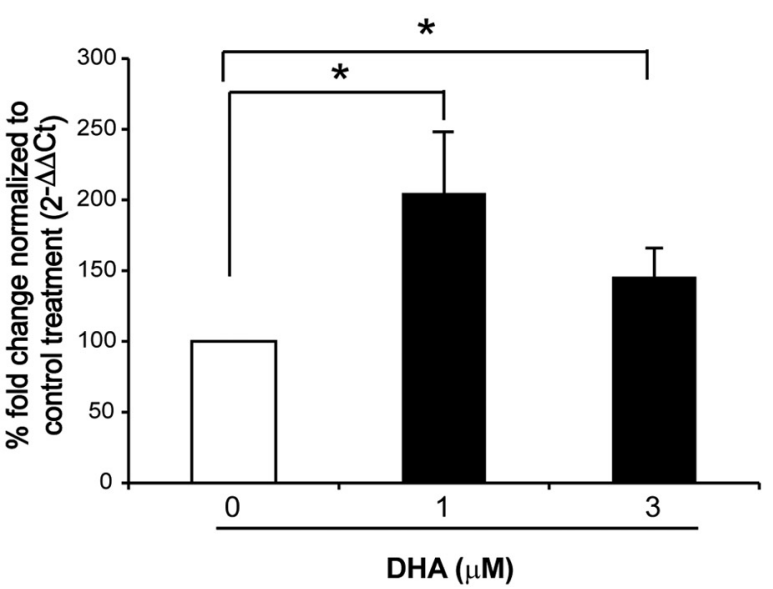

Figure 12. DHA increases miR-21 expression in vivo and in vitro. $A, B$, Low-magnification images of miR-21 ISH in rats' cerebral cortices ipsilateral to the lesion side $1 \mathrm{~d}$ following cervical SCI. $C$, $D$, Higher magnification of the dashed boxed in $A, B$ revealed stronger expression of miR-21 in layer $V$ cerebral cortex after DHA treatment. $\boldsymbol{E}$, Very low miR-21 expression in sham operated animal. $\boldsymbol{F}$, The quantification revealed that DHA significantly upregulated the level of miR-21 compared with the vehicle group. $G$, Levels of miR-21 in the cortical neuron culture were determined by qRT-PCR using the TaqMan microRNA assays. The quantitative data were expressed as fold change, in which the RNU6B acted as the endogenous control and the $0 \mu \mathrm{m}$ DHA as the control for treatment. A significant increase in miR-21 levels with 1 and $3 \mu \mathrm{m}$ DHA was observed in cortical neuron cultures at 3 DIV. ${ }^{*} p<0.05$. 
that a fish oil diet (rich in DHA) decreases PTEN (Kansal et al., 2014). Interestingly, DHA has also been shown to have the opposite effect (i.e., an increase in PTEN and decrease in pAKT expression) in cancer cells (Mandal et al., 2012; Morin et al., 2013). The reason for this dual action of DHA on the PTEN-AKT-mTOR pathway is not known, and further investigation is necessary.

Neuroplasticity can be detrimental as well as beneficial. Beneficial changes include compensatory sprouting of intact axons to take over a denervated pathway and restore lost function, and the formation of new neuronal circuits that bypass the injury site. In our behavioral assessments of lateral hemisected rats and pyramidotomy mice, we obtained evidence for a beneficial role of this DHA-promoted plasticity by demonstrating a precise and positive correlation between sprouting of uninjured CST axons and improved foot placement on the grid walk test and/or food pellet grasping in the staircase task. Both these tasks are known to be associated with CST function (Montoya et al., 1991; Metz and Whishaw, 2002). Furthermore, DHA treatment significantly increased sprouting of uninjured CST fibers within the gray matter of the ipsilateral region caudal to the lesion site. The early recovery observed in the open field locomotion test a few days after hemisection involves gross motor control and is likely due to the neuroprotective effect of DHA, as previously observed (King et al., 2006, Huang et al., 2007). However, the delayed functional improvement after DHA, which took 1 or 2 weeks to occur in the staircase test after hemisection and in the grid exploration test after pyramidotomy, involves fine motor control and likely involves neuroplasticity, in agreement with previous studies (Cafferty et al., 2008; Yip et al., 2010). These results provide support for anatomical plasticity playing a key role in the therapeutic effect of DHA. This study also revealed that DHA can promote sprouting CST axons after pyramidotomy to contact V2a interneurons, which are unilateral propriospinal interneurons (Lee and Pfaff, 2001; Al-Mosawie et al., 2007). Previous studies have demonstrated that spinal cord plasticity can result in sprouting axons making indirect reconnection to caudal targets via propriospinal interneurons (Bareyre et al., 2004; Vavrek et al., 2006) or direct reconnection onto motor neurons (Bareyre et al., 2005).

In addition to the voluntary CST pathway, DHA treatment also markedly enhanced axonal sprouting in the serotonergic pathway, which plays an essential role in modulating and triggering neuronal activity in the spinal cord (Ciranna, 2006; Jordan et al., 2008). The lateral hemisection injury increased the serotonin fiber intensity rostral to the lesion at 1 and 3 weeks after injury. Although there was reduced serotonin fiber density at 1 week after injury in the caudal part of the spinal cord, by 3 weeks after injury, serotonin immunoreactivity recovered to baseline level (Fig. $5 \mathrm{~J}$ ). The finding of a local recovery of serotonin immunoreactivity caudal to the lesion site is supported by previous investigations in thoracic spinal cord hemisection (Hains et al., 2002; Saruhashi et al., 2009; Hawthorne et al., 2011). It is notable that the serotonin axons increased in the perilesional area in the DHA-treated group, especially in the spinal cord caudal to the lesion site. The general density of serotonin fibers increased in the DHA-treated group suggests that the acute treatment of DHA can induce additional anatomical plasticity specifically through serotonergic fibers.

Another type of neuroplasticity is synaptic plasticity, which involves activity-dependent change in the strength of existing synapses, thereby improving synaptic transmission. In animal studies, orally administered DHA-containing preparations have been shown to promote the synthesis of synaptic membranes and specific presynaptic and postsynaptic proteins (Wurtman et al.,
2006) and improve brain learning associated with synaptic plasticity (Wu et al., 2008). It has been reported that polyunsaturated fatty acid supplementation is associated with an increased expression of synaptophysin in the hippocampus (Solfrizzi et al., 2006; Venna et al., 2009). At the molecular level, DHA can also affect neural function by enhancing synaptic membrane fluidity and function (Jump, 2002). Our study was not designed to identify whether sprouting or spared axons show increased synaptic transmission, but we did examine changes in synaptophysin, a synaptic vesicle protein that is present in presynaptic axons. Previous studies demonstrated that synaptophysin immunoreactivity is reduced in the spinal cord caudal to the lesion site after spinal cord hemisection or transection (Nacimiento et al., 1995; Macias et al., 2009; López-Dolado et al., 2013), which is similar to our data. The decreased global synaptophysin expression caudal to the lesion site in our vehicle-treated group was not present in DHA-treated animals. We additionally observed an increase in synaptophysin boutons contacting motor neurons in the DHAtreated cervical spinal cord, and this likely represents an enhancement of motor neuron synaptic input after DHA treatment. In cultures of embryonic cortical neurons, 1-3 $\mu \mathrm{M}$ DHA not only increased global synaptophysin expression but also increased synaptophysin boutons contacting cortical neurons and proximal neurites. These findings are consistent with previous research that demonstrated by electrophysiology methods (Cao et al., 2009) that DHA can enhance synaptic function in hippocampal neuronal development.

In conclusion, our findings indicate in two different animal models of CNS traumatic injury that DHA can improve neurological recovery by directly influencing anatomical and synaptic plasticity. Our morphological and biochemical findings suggest that DHA can enhance synaptic contacts made by sprouting axons. Furthermore, we show that the anatomical plasticity promoted by DHA is associated with a reduction in PTEN expression, an increase in pAKT, and an increase in miR-21. These results together provide direct evidence for DHA promoting neuritogenesis and suggest that this effect could be a mechanism underlying its beneficial effect on behavioral recovery. The full repertoire of molecular mechanisms involved in the effect of DHA on neuroplasticity after CNS injury deserves further investigation.

\section{References}

Akbar M, Calderon F, Wen Z, Kim HY (2005) Docosahexaenoic acid: a positive modulator of Akt signaling in neuronal survival. Proc Natl Acad Sci U S A 102:10858-10863. CrossRef Medline

Al-Mosawie A, Wilson JM, Brownstone RM (2007) Heterogeneity of V2derived interneurons in the adult mouse spinal cord. Eur J Neurosci 26: 3003-3015. CrossRef Medline

Averill S, Robson LG, Jeromin A, Priestley JV (2004) Neuronal calcium sensor- 1 is expressed by dorsal root ganglion cells, is axonally transported to central and peripheral terminals, and is concentrated at nodes. Neuroscience 123:419-427. CrossRef Medline

Azim E, Jiang J, Alstermark B, Jessell TM (2014) Skilled reaching relies on a V2a propriospinal internal copy circuit. Nature 508:357-363. CrossRef Medline

Bailes JE, Mills JD (2010) Docosahexaenoic acid reduces traumatic axonal injury in a rodent head injury model. J Neurotrauma 27:1617-1624. CrossRef Medline

Bareyre FM, Kerschensteiner M, Raineteau O, Mettenleiter TC, Weinmann O, Schwab ME (2004) The injured spinal cord spontaneously forms a new intraspinal circuit in adult rats. Nat Neurosci 7:269-277. CrossRef Medline

Bareyre FM, Kerschensteiner M, Misgeld T, Sanes JR (2005) Transgenic labeling of the corticospinal tract for monitoring axonal responses to spinal cord injury. Nat Med 11:1355-1360. CrossRef Medline 
Basso DM, Beattie MS, Bresnahan JC (1995) A sensitive and reliable locomotor rating scale for open field testing in rats. J Neurotrauma 12:1-21. CrossRef Medline

Begum G, Yan HQ, Li L, Singh A, Dixon CE, Sun D (2014) Docosahexaenoic acid reduces ER stress and abnormal protein accumulation and improves neuronal function following traumatic brain injury. J Neurosci 34:3743-3755. CrossRef Medline

Belayev L, Khoutorova L, Atkins KD, Bazan NG (2009) Robust docosahexaenoic acid-mediated neuroprotection in a rat model of transient, focal cerebral ischemia. Stroke 40:3121-3126. CrossRef Medline

Belayev L, Khoutorova L, Atkins KD, Eady TN, Hong S, Lu Y, Obenaus A, Bazan NG (2011) Docosahexaenoic acid therapy of experimental ischemic stroke. Transl Stroke Res 2:33-41. CrossRef Medline

Cafferty WB, Bradbury EJ, Lidierth M, Jones M, Duffy PJ, Pezet S, McMahon SB (2008) Chondroitinase ABC-mediated plasticity of spinal sensory function. J Neurosci 28:11998-12009. CrossRef Medline

Calderon F, Kim HY (2004) Docosahexaenoic acid promotes neurite growth in hippocampal neurons. J Neurochem 90:979-988. CrossRef Medline

Cao D, Kevala K, Kim J, Moon HS, Jun SB, Lovinger D, Kim HY (2009) Docosahexaenoic acid promotes hippocampal neuronal development and synaptic function. J Neurochem 111:510-521. CrossRef Medline

Cao Y, Shumsky JS, Sabol MA, Kushner RA, Strittmatter S, Hamers FP, Lee DH, Rabacchi SA, Murray M (2008) Nogo-66 receptor antagonist peptide (NEP1-40) administration promotes functional recovery and axonal growth after lateral funiculus injury in the adult rat. Neurorehabil Neural Repair 22:262-278. CrossRef Medline

Christie KJ, Webber CA, Martinez JA, Singh B, Zochodne DW (2010) PTEN inhibition to facilitate intrinsic regenerative outgrowth of adult peripheral axons. J Neurosci 30:9306-9315. CrossRef Medline

Ciranna L (2006) Serotonin as a modulator of glutamate- and GABAmediated neurotransmission: implications in physiological functions and in pathology. Curr Neuropharmacol 4:101-114. CrossRef Medline

Desai A, Kevala K, Kim HY (2014) Depletion of brain docosahexaenoic acid impairs recovery from traumatic brain injury. PLoS One 9:e86472. CrossRef Medline

Ding J, Guo J, Yuan Q, Yuan F, Chen H, Tian H (2013) Inhibition of phosphatase and tensin homolog deleted on chromosome 10 decreases rat cortical neuron injury and blood-brain barrier permeability, and improves neurological functional recovery in traumatic brain injury model. PLoS One 8:e80429. CrossRef Medline

Dougherty KJ, Kiehn O (2010) Functional organization of V2a-related locomotor circuits in the rodent spinal cord. Ann N Y Acad Sci 1198:85-93. CrossRef Medline

Duplus E, Glorian M, Forest C (2000) Fatty acid regulation of gene transcription. J Biol Chem 275:30749-30752. CrossRef Medline

Eady TN, Belayev L, Khoutorova L, Atkins KD, Zhang C, Bazan NG (2012) Docosahexaenoic acid signaling modulates cell survival in experimental ischemic stroke penumbra and initiates long-term repair in young and aged rats. PLoS One 7:e46151. CrossRef Medline

Edgerton VR, Tillakaratne NJ, Bigbee AJ, de Leon RD, Roy RR (2004) Plasticity of the spinal neural circuitry after injury. Annu Rev Neurosci 27: 145-167. CrossRef Medline

Figueroa JD, Cordero K, Llán MS, De Leon M (2013) Dietary omega-3 polyunsaturated fatty acids improve the neurolipidome and restore the DHA status while promoting functional recovery after experimental spinal cord injury. J Neurotrauma 30:853-868. CrossRef Medline

Fouad K, Tetzlaff W (2012) Rehabilitative training and plasticity following spinal cord injury. Exp Neurol 235:91-99. CrossRef Medline

García-Alías G, Barkhuysen S, Buckle M, Fawcett JW (2009) Chondroitinase $A B C$ treatment opens a window of opportunity for task-specific rehabilitation. Nat Neurosci 12:1145-1151. CrossRef Medline

Ge XT, Lei P, Wang HC, Zhang AL, Han ZL, Chen X, Li SH, Jiang RC, Kang CS, Zhang JN (2014) miR-21 improves the neurological outcome after traumatic brain injury in rats. Sci Rep 4:6718. CrossRef Medline

Gulino R, Dimartino M, Casabona A, Lombardo SA, PerciavalleV (2007) Synaptic plasticity modulates the spontaneous recovery of locomotion after spinal cord hemisection. Neurosci Res 57:148-156. CrossRef Medline

Hains BC, Everhart AW, Fullwood SD, Hulsebosch CE (2002) Changes in serotonin, serotonin transporter expression and serotonin denervation supersensitivity: involvement in chronic central pain after spinal hemisection in the rat. Exp Neurol 175:347-362. CrossRef Medline
Han Z, Chen F, Ge X, Tan J, Lei P, Zhang J (2014) miR-21 alleviated apoptosis of cortical neurons through promoting PTEN-Akt signaling pathway in vitro after experimental traumatic brain injury. Brain Res 1582: 12-20. CrossRef Medline

Hashimoto M, Hossain S, Shimada T, Sugioka K, Yamasaki H, Fujii Y, Ishibashi Y, Oka J, Shido O (2002) Docosahexaenoic acid provides protection from impairment of learning ability in Alzheimer's disease model rats. J Neurochem 81:1084-1091. CrossRef Medline

Hawthorne AL, Hu H, Kundu B, Steinmetz MP, Wylie CJ, Deneris ES, Silver J (2011) The unusual response of serotonergic neurons after CNS injury: lack of axonal dieback and enhanced sprouting within the inhibitory environment of the glial scar. J Neurosci 31:5605-5616. CrossRef Medline

Hu JZ, Huang JH, Zeng L, Wang G, Cao M, Lu HB (2013) Anti-apoptotic effect of microRNA-21 after contusion spinal cord injury in rats. J Neurotrauma 30:1349-1360. CrossRef Medline

Huang WL, King VR, Curran OE, Dyall SC, Ward RE, Lal N, Priestley JV, Michael-Titus AT (2007) A combination of intravenous and dietary docosahexaenoic acid significantly improves outcome after spinal cord injury. Brain 130:3004-3019. CrossRef Medline

Ichiyama RM, Courtine G, Gerasimenko YP, Yang GJ, van den Brand R, Lavrov IA, Zhong H, Roy RR, Edgerton VR (2008) Step training reinforces specific spinal locomotor circuitry in adult spinal rats. J Neurosci 28:7370-7375. CrossRef Medline

Jordan LM, Liu J, Hedlund PB, Akay T, Pearson KG (2008) Descending command systems for the initiation of locomotion in mammals. Brain Res Rev 57:183-191. CrossRef Medline

Jump DB (2002) The biochemistry of $\mathrm{n}-3$ polyunsaturated fatty acids. J Biol Chem 277:8755-8758. CrossRef Medline

Kansal S, Bhatnagar A, Agnihotri N (2014) Fish oil suppresses cell growth and metastatic potential by regulating PTEN and NF-kappaB signaling in colorectal cancer. PLoS One 9:e84627. CrossRef Medline

King VR, Huang WL, Dyall SC, Curran OE, Priestley JV, Michael-Titus AT (2006) Omega-3 fatty acids improve recovery, whereas omega- 6 fatty acids worsen outcome, after spinal cord injury in the adult rat. J Neurosci 26:4672-4680. CrossRef Medline

Lee SK, Pfaff SL (2001) Transcriptional networks regulating neuronal identity in the developing spinal cord. Nat Neurosci 4 [Suppl]:1183-1191.

Lim GP, Calon F, Morihara T, Yang F, Teter B, Ubeda O, Salem N Jr, Frautschy SA, Cole GM (2005) A diet enriched with the omega-3 fatty acid docosahexaenoic acid reduces amyloid burden in an aged Alzheimer mouse model. J Neurosci 25:3032-3040. CrossRef Medline

Lim SN, Huang W, Hall JC, Michael-Titus AT, Priestley JV (2013) Improved outcome after spinal cord compression injury in mice treated with docosahexaenoic acid. Exp Neurol 239:13-27. CrossRef Medline

Liu G, Detloff MR, Miller KN, Santi L, Houlé JD (2012) Exercise modulates microRNAs that affect the PTEN/mTOR pathway in rats after spinal cord injury. Exp Neurol 233:447-456. CrossRef Medline

Liu K, Lu Y, Lee JK, Samara R, Willenberg R, Sears-Kraxberger I, Tedeschi A, Park KK, Jin D, Cai B, Xu B, Connolly L, Steward O, Zheng B, He Z (2010) PTEN deletion enhances the regenerative ability of adult corticospinal neurons. Nat Neurosci 13:1075-1081. CrossRef Medline

López-Dolado E, Lucas-Osma AM, Collazos-Castro JE (2013) Dynamic motor compensations with permanent, focal loss of forelimb force after cervical spinal cord injury. J Neurotrauma 30:191-210. CrossRef Medline

Lopez-Ramirez MA, Wu D, Pryce G, Simpson JE, Reijerkerk A, King-Robson J, Kay O, de Vries HE, Hirst MC, Sharrack B, Baker D, Male DK, Michael GJ, Romero IA (2014) MicroRNA-155 negatively affects blood-brain barrier function during neuroinflammation. FASEB J 28:2551-2565. CrossRef Medline

Macias M, Nowicka D, Czupryn A, Sulejczak D, Skup M, Skangiel-Kramska J, Czarkowska-Bauch J (2009) Exercise-induced motor improvement after complete spinal cord transection and its relation to expression of brain-derived neurotrophic factor and presynaptic markers. BMC Neurosci 10:144. CrossRef Medline

Mandal CC, Ghosh-Choudhury T, Dey N, Choudhury GG, GhoshChoudhury N (2012) miR-21 is targeted by omega-3 polyunsaturated fatty acid to regulate breast tumor CSF-1 expression. Carcinogenesis 33: 1897-1908. CrossRef Medline

Metz GA, Whishaw IQ (2002) Cortical and subcortical lesions impair skilled walking in the ladder rung walking test: a new task to evaluate foreand hindlimb stepping, placing, and co-ordination. J Neurosci Methods 115:169-179. CrossRef Medline 
Montoya CP, Campbell-Hope LJ, Pemberton KD, Dunnett SB (1991) The "staircase test": a measure of independent forelimb reaching and grasping abilities in rats. J Neurosci Methods 36:219-228. CrossRef Medline

Morin C, Fortin S, Cantin AM, Sirois M, Sirois C, Rizcallah E, Rousseau É (2013) Anti-cancer effects of a new docosahexaenoic acid monoacylglyceride in lung adenocarcinoma. Recent Pat Anticancer Drug Discov 8:319-334. CrossRef Medline

Nacimiento W, Sappok T, Brook GA, Tóth L, Schoen SW, Noth J, Kreutzberg GW (1995) Structural changes of anterior horn neurons and their synaptic input caudal to a low thoracic spinal cord hemisection in the adult rat: a light and electron microscopic study. Acta Neuropathol 90:552-564. CrossRef Medline

Nieto-Diaz M, Esteban FJ, Reigada D, Muñoz-Galdeano T, Yunta M, Caballero-López M, Navarro-Ruiz R, Del Águila A, Maza RM (2014) MicroRNA dysregulation in spinal cord injury: causes, consequences and therapeutics. Front Cell Neurosci 8:53. Medline

Pan HC, Kao TK, Ou YC, Yang DY, Yen YJ, Wang CC, Chuang YH, Liao SL, Raung SL, Wu CW, Chiang AN, Chen CJ (2009) Protective effect of docosahexaenoic acid against brain injury in ischemic rats. J Nutr Biochem 20:715-725. CrossRef Medline

Park KK, Liu K, Hu Y, Smith PD, Wang C, Cai B, Xu B, Connolly L, Kramvis I, Sahin M, He Z (2008) Promoting axon regeneration in the adult CNS by modulation of the PTEN/mTOR pathway. Science 322:963-966. CrossRef Medline

Paterniti I, Impellizzeri D, Di Paola R, Esposito E, Gladman S, Yip P, Priestley JV, Michael-Titus AT, Cuzzocrea S (2014) Docosahexaenoic acid attenuates the early inflammatory response following spinal cord injury in mice: in-vivo and in-vitro studies. J Neuroinflammation 11:6. CrossRef Medline

Puskás LG, Kitajka K, Nyakas C, Barcelo-Coblijn G, Farkas T (2003) Shortterm administration of omega 3 fatty acids from fish oil results in increased transthyretin transcription in old rat hippocampus. Proc Natl Acad Sci U S A 100:1580-1585. CrossRef Medline

Raineteau O, Schwab ME (2001) Plasticity of motor systems after incomplete spinal cord injury. Nat Rev Neurosci 2:263-273. CrossRef Medline

Ramer LM, Borisoff JF, Ramer MS (2004) Rho-kinase inhibition enhances axonal plasticity and attenuates cold hyperalgesia after dorsal rhizotomy. J Neurosci 24:10796-10805. CrossRef Medline

Robson LG, Dyall S, Sidloff D, Michael-Titus AT (2010) Omega-3 polyunsaturated fatty acids increase the neurite outgrowth of rat sensory neurones throughout development and in aged animals. Neurobiol Aging 31:678-687. CrossRef Medline

Russell KL, Berman NE, Levant B (2013) Low brain DHA content worsens sensorimotor outcomes after TBI and decreases TBI-induced Timp1 expression in juvenile rats. Prostaglandins Leukot Essent Fatty Acids 89:97105. CrossRef Medline

Salem N Jr, Litman B, Kim HY, Gawrisch K (2001) Mechanisms of action of docosahexaenoic acid in the nervous system. Lipids 36:945-959. CrossRef Medline

Sandhir R, Gregory E, Berman NE (2014) Differential response of miRNA-21 and its targets after traumatic brain injury in aging mice. Neurochemistry Int 78:117-121. CrossRef Medline

Saruhashi Y, Matsusue Y, Fujimiya M (2009) The recovery of 5-HT trans- porter and 5-HT immunoreactivity in injured rat spinal cord. Arch Orthop Trauma Surg 129:1279-1285. CrossRef Medline

Solfrizzi V, Colacicco AM, D'Introno A, Capurso C, Torres F, Rizzo C, Capurso A, Panza F (2006) Dietary intake of unsaturated fatty acids and age-related cognitive decline: a 8.5-year follow-up of the Italian Longitudinal Study on Aging. Neurobiology Aging 27:1694-1704. CrossRef Medline

Starkey ML, Barritt AW, Yip PK, Davies M, Hamers FP, McMahon SB, Bradbury EJ (2005) Assessing behavioural function following a pyramidotomy lesion of the corticospinal tract in adult mice. Exp Neurol 195: 524-539. CrossRef Medline

Strickland IT, Richards L, Holmes FE, Wynick D, Uney JB, Wong LF (2011) Axotomy-induced miR-21 promotes axon growth in adult dorsal root ganglion neurons. PLoS One 6:e23423. CrossRef Medline

Tetzlaff W, Fouad K, Kwon B (2009) Be careful what you train for. Nat Neurosci 12:1077-1079. CrossRef Medline

Vavrek R, Girgis J, Tetzlaff W, Hiebert GW, Fouad K (2006) BDNF promotes connections of corticospinal neurons onto spared descending interneurons in spinal cord injured rats. Brain 129:1534-1545. CrossRef Medline

Venna VR, Deplanque D, Allet C, Belarbi K, Hamdane M, Bordet R (2009) PUFA induce antidepressant-like effects in parallel to structural and molecular changes in the hippocampus. Psychoneuroendocrinology 34:199211. CrossRef Medline

Wang D, Sun T (2011) Neural plasticity and functional recovery of human central nervous system with special reference to spinal cord injury. Spinal Cord 49:486-492. CrossRef Medline

Ward RE, Huang W, Curran OE, Priestley JV, Michael-Titus AT (2010) Docosahexaenoic acid prevents white matter damage after spinal cord injury. J Neurotrauma 27:1769-1780. CrossRef Medline

Wu A, Ying Z, Gomez-Pinilla F (2004) Dietary omega-3 fatty acids normalize BDNF levels, reduce oxidative damage, and counteract learning disability after traumatic brain injury in rats. J Neurotrauma 21:1457-1467. CrossRef Medline

Wu A, Ying Z, Gomez-Pinilla F (2008) Docosahexaenoic acid dietary supplementation enhances the effects of exercise on synaptic plasticity and cognition. Neuroscience 155:751-759. CrossRef Medline

Wurtman RJ, Ulus IH, Cansev M, Watkins CJ, Wang L, Marzloff G (2006) Synaptic proteins and phospholipids are increased in gerbil brain by administering uridine plus docosahexaenoic acid orally. Brain Res 1088: 83-92. CrossRef Medline

Yip PK, Wong LF, Pattinson D, Battaglia A, Grist J, Bradbury EJ, Maden M, McMahon SB, Mazarakis ND (2006) Lentiviral vector expressing retinoic acid receptor beta2 promotes recovery of function after corticospinal tract injury in the adult rat spinal cord. Hum Mol Genet 15:3107-3118. CrossRef Medline

Yip PK, Wong LF, Sears TA, Yáñez-Muñoz RJ, McMahon SB (2010) Cortical overexpression of neuronal calcium sensor-1 induces functional plasticity in spinal cord following unilateral pyramidal tract injury in rat. PLoS Biol 8:e1000399. CrossRef Medline

Zhao J, Qu Y, Wu J, Cao M, Ferriero DM, Zhang L, Mu D (2013) PTEN inhibition prevents rat cortical neuron injury after hypoxia-ischemia. Neuroscience 238:242-251. CrossRef Medline 